\title{
Near-inertial dissipation due to stratified flow over abyssal topography
}

By Varvara E. Zemskova (barbara.zemskova@utoronto.ca) and

Nicolas Grisouard (nicolas.grisouard@utoronto.ca)

University of Toronto, Department of Physics, 60 St. George Street, Toronto ON M5S 1A7, Canada

This paper is a non-peer reviewed preprint submitted to EarthArXiv. It was submitted to Journal of Physical Oceanography on 11 January, 2021 and is currently undergoing peer review.

Please feel free to reach out to us if you have any questions or comments regarding the paper. 


\title{
Near-inertial dissipation due to stratified flow over abyssal topography
}

\author{
Varvara E. Zemskova* and Nicolas Grisouard
}

Department of Physics, University of Toronto, Toronto, ON, Canada

${ }_{4}{ }^{*}$ Corresponding author: Varvara E. Zemskova, barbara.zemskova@ utoronto.ca 


\section{ABSTRACT}

${ }_{5}$ Linear theory for steady stratified flow over topography sets the range for topographic wavenumbers

6

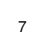

over which freely propagating internal waves are generated, and the radiation and breaking of these waves contribute to energy dissipation away from the ocean bottom. However, previous numerical work demonstrated that dissipation rates can be enhanced by flow over large scale topographies with wavenumbers outside of the lee wave radiative range. We conduct idealized 3D numerical simulations of steady stratified flow over 1D topography in a rotating domain and quantify vertical distribution of kinetic energy dissipation. We vary two parameters: the first determines whether the topographic obstacle is within the lee wave radiative range and the second, proportional to the topographic height, measures the degree of flow non-linearity. For a certain combination of topographic width and height, the flow develops periodicity in wave breaking and kinetic energy dissipation; in these simulations, kinetic energy dissipation rates are also enhanced in the interior of the domain. In the radiative regime the inertial motions arise due to resonant wavewave interactions. In the small wavenumber non-radiative regime, instabilities downstream of the obstacle can facilitate the generation and propagation of non-linearly forced inertial motions, especially as topographic height increase. In our simulations, dissipation rates for tall and wide non-radiative topography are comparable to those of radiative topography, even away from the bottom, which is relevant to the ocean where the topographic spectrum is such that wider abyssal hills also tend to be taller. 


\section{Introduction}

Small-scale turbulence plays an important role in dissipating energy input from winds, tides, and surface buoyancy forcing (Munk and Wunsch 1998; Wunsch and Ferrari 2004; Hughes et al. 2009; Zemskova et al. 2015) and in subsequently sustaining the meridional overturning circulation through diapycnal mixing (Marshall and Speer 2012; Talley 2013). The background diffusivities in the ocean are generally too small to maintain the stratification; however, the diffusivity values have a high degree of spatial variability and mixing occurs in certain hot spots (De Lavergne et al. 2016; Mashayek et al. 2017). One of the identified important routes for energy dissipation in the ocean interior is the breaking of the internal waves that are generated as a result of the steady geostrophic, eddy, or tidal flows impinging on rough bottom topography. Many observational (e.g., St. Laurent et al. 2012; Sheen et al. 2013; Waterman et al. 2013; Brearley et al. 2013; Cusack et al. 2020) and numerical (e.g., Nikurashin and Ferrari 2010a; Nikurashin et al. 2014; Yang et al. 2018) studies of this energy pathway have focused on the Southern Ocean. There, the circulation is driven by both the strong persistent zonal winds and surface buoyancy forcing. However, enhanced dissipation rates overlying rough topography are ubiquitous in other regions of the ocean as well (e.g. Liang and Thurnherr 2012; Whalen et al. 2012; Musgrave et al. 2017; Hu et al. 2020).

Global and regional ocean models often do not have sufficient resolution to capture small-scale rough topographic features, and the internal motions generated by the flow over them have to be parameterized. To our knowledge, most parameterizations include effects of lee wave radiation, but neglect to include non-radiative, non-linear hydraulic effects. In this work, we illustrate the interplay between the two in a series of idealized numerical simulations.

In a regime we hereafter refer to as "radiative", when a steady, homogeneous flow with velocity $U$ and buoyancy frequency $N$ goes over a sinusoidal bottom topography with wavenumber $k$, freely 
propagating linear lee waves are generated if

$$
\chi=\frac{U k}{N} \in\left(\frac{|f|}{N}, 1\right)
$$

where $f$ is the local Coriolis parameter (e.g., Bell 1975; Nikurashin and Ferrari 2010b). The energy transport and upward momentum flux of these lee waves can then be computed according to Eliassen (1960). For the value of $U$ and $N$ observed in the Drake Passage, this constraint sets the range of radiative topographic wavelengths to approximately $500 \mathrm{~m}$ to $5 \mathrm{~km}$ (St. Laurent et al. 2012).

Another important non-dimensional number defined in Nikurashin and Ferrari (2010b) is the steepness parameter (or inverse Froude number):

$$
J=\frac{N h_{0}}{U},
$$

where $h_{0}$ is the topographic height. When $J \ll 1$, the topographic regime is called subcritical and the waves are linear. However, in the supercritical $J>1$ regime, the linear theory is no longer valid, as the non-linear effects, such as upstream blocking and downstream hydraulic control become important (Klymak et al. 2010; Winters and Armi 2012). Mayer and Fringer (2017) also identified $J$ as the lee wave Froude number, noting that as $J \rightarrow 1$, the height of the topography approaches the vertical scale of the lee wave. While linear and non-linear regimes occur over a continuous spectrum, for brevity, we refer to $J<1$ as the linear and $J>1$ as the non-linear regimes throughout the paper.

Near topography, linear internal wave theory may not hold, as Cusack et al. (2020) recently showed with energy transfer calculations using mooring array data in the Southern Ocean. In order to account for these non-linearities, Nikurashin and Ferrari (2010b) introduced an empiricallyderived correction parameter for the energy conversion rate from the background flow into the lee 
waves above a certain value of $J$, which was later adjusted for 3D topographic effects by Nikurashin et al. (2014).

For flows over topographies with non-radiative wavelengths, waves are evanescent per linear stability theory. To our knowledge, the regime in which rotation is included and the topographic wavelength is shorter than the shortest radiative wavelength, and which we touch upon in this article, has not been investigated. On the other side of the topographic spectrum, however, numerical simulations by Klymak (2018) demonstrated that flow over topography longer than radiative wavelengths (i.e., $\chi<|f| / N$ ) could generate dissipation rates even higher than the flow over the radiative topography. The author used the same topographic spectrum as Nikurashin et al. (2014), which employs the statistical model for bathymetry by Goff and Jordan (1988) with parameters fit to the Drake Passage observational data. This spectrum is red; that is, large-scale topographic features also have greater heights $h_{0}$ compared with the heights of the radiative range topography, such that in those regimes $J \gg 1$.

Another recent numerical study by Mayer and Fringer (2020) investigated the combined effects of $(\chi, J)$ on lee wave drag in flows over sinusoidal topography, albeit without rotation. The authors found that at large $\chi$, even within the radiative regime, lee wave drag is reduced compared to the linear lee wave theoretical predictions. In their study, they identify two mechanisms, "evanescent masking" and "evanescent undulations," to explain the resulting absence of radiated lee waves. These mechanisms play a role when the flow develops a blocked layer and the lowest overtopping streamline (LOTS) becomes separated from the topography. The path of the flow does not follow the bathymetry anymore, and the LOTS becomes the effective topography. As a result, the portion of the LOTS that is approximately parallel to the topography is shorter than the bathymetry, further increasing the effective $\chi$ (evanescent masking). In addition, the LOTS can develop horizontal fluctuations that can act as independent bathymetry with even shorter wavelength (evanescent 
undulation). Hence, the results from both Klymak (2018) and Mayer and Fringer (2020) suggest that there may be differences in the flow characteristics and energetics between the high and low wavenumber non-radiative regimes, which we investigate in this study.

Nikurashin and Ferrari (2010b) found that for flows over radiative topography with $J>0.4$, there is a resonant feedback between the background mean flow and the inertial oscillations that develop near the topography due to the lee wave radiation and dissipation. Because such inertial oscillations tend to have high vertical wavenumbers, they then further enhance the wave breaking, dissipation, and the mixing rate away from the topography.

However, inertial and near-inertial waves can arise whenever the geostrophic balance of the flow is lost (Vanneste 2013; Alford et al. 2016) and, for example, due to resonant and non-resonant non-linear interactions among the oceanic internal waves (Lvov et al. 2012). The loss of balance near the topography can occur due to forced vertical motion of otherwise-balanced motion over topographic obstacles and the non-linear interactions that result from the asymmetric acceleration of the flow downstream of the obstacle, akin to hydraulic control. As such, we investigate the roles that the topographic height and width play in facilitating these non-linear interactions and generation inertial motions.

The goal of this paper is to include several of these considerations to extend the Nikurashin and Ferrari (2010b) idealized simulations of steady flows over cross-stream-invariant topography. We explore the parameter regime along two dimensions, namely, that of topographic regimes outside of the radiative range with $\chi$, and that of the degree of non-linearity with $J$. We include rotation in our simulations, as $N$ is small and approaches $f$ in a large portion of the abyssal ocean (Kunze and Lien 2019). While our simulation domain is 3D, the 1D topography does not allow for the flow to go around the obstacle. Nevertheless, this set-up allows for the direct comparison with the linear lee wave theory, the results are further applicable to flows over long and wide ridges (e.g., 
Legg and Klymak 2008; Liang and Thurnherr 2012), and because turbulent processes are allowed to develop in $3 \mathrm{D}$, the dissipation mechanisms are somewhat plausible.

This process study of bottom-radiated energy propagation primarily focuses on the kinetic energy dissipation rates near the topography and in the interior of the domain. We describe the set up of our idealized simulations in different topographic regimes in $\$ 2$ and observe that for some of the simulations, there is an inertial periodicity in kinetic energy dissipation (§3) and that these simulations also have greater kinetic energy dissipation rates. We then further explore (i) whether inertial oscillations arise for the non-radiative topographies (§4) and (ii) where the nonlinear interactions with such inertial motions occur for different topographies (§5). We find that the inertial periodicity of kinetic energy dissipation develops only in the simulations, in which both inertial oscillations and time-mean flow (either in a form of freely propagating lee waves or non-linearly forced flow) are present. Furthermore, as we relate in $\S 6$, dissipation rates are enhanced with stronger non-linear interactions between the inertial oscillations and the time-mean flow. These dynamics are correlated with the combination of $\chi$ and $J$, rather than either of the parameters alone. Finally, in $\S 7$ we connect our findings to the ocean dynamics, emphasizing the distinctions between the wide and narrow non-radiative topographies.

\section{Model set-up}

We solve non-hydrostatic Navier-Stokes equations with added rotation in the Boussinesq approximation, namely,

$$
\begin{array}{r}
\frac{\partial \hat{\mathbf{u}}}{\partial t}+\hat{\mathbf{u}} \cdot \nabla \hat{\mathbf{u}}+\hat{f} \mathbf{k} \times \hat{\mathbf{u}}=-\frac{\nabla \hat{p}}{\rho_{0}}+\hat{b} \mathbf{k}+\hat{v} \nabla^{2} \hat{\mathbf{u}}+\hat{f} \hat{U} \mathbf{j}, \\
\frac{\partial \hat{b}}{\partial t}+\hat{\mathbf{u}} \cdot \nabla \hat{b}=\hat{\kappa} \nabla^{2} \hat{b} \quad \text { and } \quad \nabla \cdot \hat{\mathbf{u}}=0
\end{array}
$$

where $\hat{\mathbf{u}}=(\hat{u}, \hat{v}, \hat{w})$ is velocity in Cartesian directions $(x, y, z)$ with $z$ pointing upward, $\hat{b}=-\hat{g}(\hat{\rho}-$ $\left.\hat{\rho_{0}}\right) / \rho_{0}$ is buoyancy, with $\hat{\rho}$ the density and $\hat{\rho_{0}}$ a constant reference density, $\hat{p}$ is pressure, $\mathbf{j}, \mathbf{k}$ are the 
along-ridge and vertical unit vectors, respectively, $\hat{U}$ is a constant, cross-ridge geostrophic velocity we prescribe, $\hat{v}$ is kinematic viscosity, and $\hat{\kappa}$ is diffusivity $(\hat{\bullet}$ here represent dimensional quantities). We apply a body force of $\hat{f} \hat{U}$ to the $y$-momentum equation. This body force represents barotropic pressure gradient that is geostrophically balanced by the mean flow at all depths (Nikurashin and Ferrari 2010b; Klymak 2018).

We solve Eqns. (3) using Nek5000, a spectral-element code (Fischer et al. 2008) that has been previously used in many studies of stratified flows (e.g., Özgökmen et al. 2004; Mashayek and Peltier 2012; Salehipour et al. 2015; Fabregat Tomàs et al. 2016; Ezhova et al. 2018). It permits the implementation of bottom topography, offers flexibility over mesh size, and combines the traditional advantages of pseudo-spectral methods, such as computational speed and accuracy. We use it to run a DNS formulation of the Navier-Stokes equations, which resolves fluid motions from the basin scales to the smallest spatio-temporal dissipative scales allowed by our mesh resolution without employing subgrid turbulence parameterization or filtering and allows us to compute viscous dissipation directly.

We run simulations in a highly idealized rectangular domain shown in Fig. 1. All physical variables are non-dimensionalized using ocean depth $\hat{H}$ for length scales, $1 / \hat{f}$ for time scales, and $\Delta \hat{\rho} \hat{H}^{3}$ for mass, where $\Delta \hat{\rho}$ is fluid density difference between the surface and the bottom of the domain. All variables quantities lose their hats upon non-dimensionalization (e.g., $\hat{u} /(\hat{H} \hat{f})=u$, $\hat{f} / \hat{f}=f=1, v=\hat{v} /\left(\hat{H}^{2} \hat{f}\right)$ ). The simulation domain is doubly-periodic in $x$ (cross-ridge) and $y$ (along-ridge) directions. The bottom boundary is no-slip, with a bottom height defined by

$$
h(x)=h_{0} \sin ^{2}(k x / 2),
$$

where $h_{0}$ is the maximum topographic height (see Fig. 1). The horizontal extent of the domain is $L_{x}=2 \pi / k$, while $L_{y}$ varies with $k$ for computational efficiency. The top surface is a rigid lid with 
no-buoyancy-flux and no-slip boundary conditions, and the vertical extent of the domain varies with $k$ and is taken sufficiently large to avoid reflections from the surface to influence our results. The sizes of the computational domains for each simulation are summarized in Table 1. For all the simulations, we set Prandtl number $\operatorname{Pr}=v / \kappa=1$. We also define Reynolds number as $\operatorname{Re}=U h_{0} / v$ following Winters and Armi (2012), which ranges from 625 - 2100. In dimensional terms, the viscosity and diffusivity values satisfy the threshold set by Shakespeare and Hogg (2017) (i.e., $<10^{-2} \mathrm{~m}^{2} \mathrm{~s}^{-1}$ ), but our Re is larger than in other studies that use larger scale models (e.g., Klymak 2018).

We explore the sensitivity of the near-inertial wave radiation and flow dynamics to the topography and the background flow by changing the two non-dimensional parameters that characterize the dynamical regimes as discussed in Nikurashin and Ferrari (2010b): (non-)radiative regime parameter $\chi$ (cf. Eqn. 1) and inverse Froude number $J$ (cf. Eqn. 2). Combining these two parameters leads to an expression of the bulk topographic slope

$$
\xi=\frac{h_{0}}{L_{x} / 2}=\frac{k h_{0}}{\pi}=\frac{\chi J}{\pi} .
$$

For all experiments, we fix $|f| / N=0.1$ so that the radiative (lee wave) regime corresponds to $0.1<\chi<1$. The value for $|f| / N$ corresponds to previous values of $\hat{N} \approx 10^{-3} \mathrm{~s}^{-1}$ reported from measurements in the Southern Ocean (e.g., Waterman et al. 2013) and the Coriolis parameter value at $45^{\circ} \mathrm{S}$ of $\hat{f}=-10^{-4} \mathrm{~s}^{-1}$. The regime $\chi<0.1$ represents either long wavelength topography, weak background flow and/or strong stratification, and $\chi>1$ represents either short wavelength topography, strong background flow and/or weak stratification. We hold the values for $U, f$, and $N$ fixed and vary $\chi$ and $J$ via the topographic wavenumber $k$ and height $h_{0}$, respectively, such that $\chi$ will take three values: $0.063,0.16$ and 1.12 . Throughout the text, we will refer to the simulations in the non-radiative regime with $\chi=0.063<|f| / N$ as the "wide" topography, and 
the ones with $\chi=1.12$ as the "narrow" topography, such that the width is relative to that of the radiative topography with $\chi=0.16$.

We vary the inverse Froude number $J$ over $[0.4,5]$ in order to capture the transition from linear to non-linear regime, which is the focus of this paper aligned with the topographic heights and background velocity values obtained as a part of the Diapycnal and Isopycnal Mixing Experiment in the Southern Ocean (St. Laurent et al. 2012; Waterman et al. 2013) and used in other Southern Ocean studies (e.g., Nikurashin et al. 2014; Klymak 2018). This range contrasts with the large $J=O(10-100)$ of highly non-linear regimes, which correspond to greater topographic height, weaker background flow velocity and/or stronger stratification, investigated in previous studies of stratified flows over topography (e.g. Klymak et al. 2010; Jagannathan et al. 2020).

We show the non-dimensional parameters for each numerical experiment in black dots in Figure 2 along with with the domain size and resolution summarized in Table 1. In Nek5000, grid lines along a horizontal $(x, y)$ plane follow the topographic feature; hence, the grid spacing is non-isotropic. We chose not to include simulations with short wavelength topography (small $k$, large $\chi$ ) and large inverse Froude number $J>1$, as these correspond to large slope topographies $(\xi>0.4)$ and result in a significantly anisotropic numerical grid, which would require more spatial resolution (Fischer 1997). Furthermore, the topographic spectrum is red: for example, Klymak (2018) estimated that the radiative topography in the Drake Passage has $J \approx 0.8$, while the low wavenumber wide non-radiative topography has $J>3$. To account for this low probability to find a high- $\chi$, high- $J$ topography in the ocean, we chose lower heights $(J \in[0.4,1])$ for narrow features $(\chi=1.12)$ and larger heights $(J \in[0.6,5])$ for wide features $(\chi=0.063)$. In the radiative topography $(\chi=0.16)$, we chose $J \in[0.6,2]$. We based our grid resolution $\left(\Delta_{x}, \Delta_{y}, \Delta_{z}\right)$ on the Kolmogorov microscale $\eta_{K}=\left(v^{3} / \epsilon\right)^{1 / 4}$, where $\epsilon$ is the local kinetic energy dissipation, to ensure that $\left[\Delta_{x}, \Delta_{y}, \Delta_{z}\right]_{\max }<\pi \eta_{K}$ (cf. Table 1). It is widely considered that this condition ensures the 
resolution of the smallest scales of motion, up to dissipative scales and therefore ensures a reliable DNS output, as discussed in Salehipour et al. (2015) and Gayen et al. (2014). We initialize the simulations with a uniform velocity $U$ in the cross-ridge direction ${ }^{1}$ and $B(z)$ and run them until $t=50$, corresponding to approximately 8 inertial periods, the latter being defined as $t_{I}=2 \pi /|f|$. We ran two of the simulations, namely $(\chi, J)=(0.16,0.6)$ and $(\chi, J)=(1.12,0.4)$, to $t=12 t_{I}$ to allow for at least 6 breaking events that occur every $t_{I}$. Indeed, they begin to develop later for these parameters, as we discuss in $\S 3$.

While simulations in previous studies (e.g., Nikurashin and Ferrari 2010b; Klymak et al. 2010) were ran for longer $\left(\approx 10-44 t_{I}\right)$ to study the long-term evolution of the flow, we found that a short duration $(\sim 4 /|f|)$ was sufficient to establish a quasi-steady flow, similar to the simulations of Winters and Armi (2012). We also decided not to implement the restoration term for the stratification of Nikurashin and Ferrari (2010b). However, we conducted a baseline simulation with a restoration term to compare with the simulation without the restoration term, and the results were qualitatively similar (see Appendix A for more details). For our following analysis, we consider a temporal average over the last 4 inertial periods.

\section{Kinetic Energy Dissipation}

The instantaneous normalized perturbation velocity $u / U$ field (shown in color in Figure 1) displays two features that are the focus of this paper: (i) internal waves radiating upward from the topography; and (ii) asymmetry in $u$-velocity between the upstream and downstream side of the topographic bump, which is a characteristic of hydraulic control (Winters and Armi 2012), best visible for $z<0.15$. The two prominent wave types in the internal wave field are the horizontal bands corresponding to the near-inertial oscillations and oblique bands corresponding to upward-

${ }^{1}$ This initial condition then immediately adjusts to the no-slip boundary condition at the bottom. 
propagating waves, mostly lee (oscillating with zero frequency in the topographic reference frame) and inertial waves. Throughout the paper, we will explore the interaction between the zerofrequency time-mean flow (freely propagating lee waves or otherwise forced flow) and the inertial waves in different topographic regimes.

In addition, on the downstream side, breaking occurs as indicated by isopycnal contours shown in black in Fig. 1. Remarkably, in several of our simulations, breaking events occur every $t_{l}$. Figure 1 shows the temporal evolution of the flow over one inertial period at $0.25 t_{I}$ interval highlighting such periodic breaking. We observe the flow accelerating downstream of the topography (Fig. 1(a, b)), then breaking (Fig. 1(c)), followed by an inertial wave radiating away (Fig. 1(d)). In this section, we first focus on the spatial and temporal profiles of kinetic energy (KE) dissipation in different topographic regimes with these two features in mind. The results presented in this section are used as the motivation for the detailed analysis of the flow in the subsequent sections.

In this study, we focus on KE dissipation here, as it primarily relates to energy propagation into the interior and mixing. For the velocity field $\mathbf{u}(x, y, z, t)=(u+U, v, w)$, the total kinetic energy divided by $\rho_{0}$, is

$$
E_{K}=\left[(u+U)^{2}+v^{2}+w^{2}\right] / 2 .
$$

The dissipation rate of the kinetic energy averaged in $y$-direction and in time is defined as

$$
D\left(E_{K}\right)=v|\nabla(\mathbf{u}-U \mathbf{i})|^{2},
$$

where $\mathbf{i}$ is the unit vector in $x$ direction.

Notably, with the exception of two simulations, near-bottom dissipation events, which are followed by upward propagating dissipating structures, occur every inertial period $t_{I}$. We first show this in Figure 3, with the Hovmöller diagram of the normalized total KE dissipation $D\left(E_{K}\right) / U^{2}$ for all of the simulations, horizontally averaged using the height above the bottom coordinates, 
i.e., $\mathrm{HAB}=z-h(x)$. The horizontal average is performed after we first compute $D\left(E_{K}\right)$ over the entire volume. The presence of such inertial modulation is quantified by the peak at $\omega / f=1$ in the temporal spectra of $D\left(E_{K}\right) / U^{2}$ shown in Figure $4(\mathrm{a}, \mathrm{b})$ computed at $\mathrm{HAB}=0.1,0.2$, respectively. The only two cases that do not exhibit periodicity in $D\left(E_{K}\right)$ correspond to either wide and short topography $(\chi=0.063, J=0.6$, Fig. 3(a)) or narrow and tall topography $(\chi=1.12, J=1$, Fig. 3(i)). For all of the other simulations, there is an inertial peak in the $D\left(E_{K}\right)$ spectra (denoted as $\phi_{D K E}^{t}$ ) at $\mathrm{HAB}=0.1$. At $\mathrm{HAB}=0.2$, the same happens with the exception of the $\chi=0.063, J=2$ simulation, which does not feature an inertial peak either. For topographies with $\chi<1$, the periodicity develops earlier and features a stronger signature of the upward propagating dissipation at higher values of $J$. In contrast, the inertial modulation signature is the strongest at $J=0.6$ for $\chi=1.12$, compared with $J=0.4$ and $J=0.3$ (not shown).

For comparisons of $D\left(E_{K}\right) / U^{2}$ as a function of HAB with respect to non-linearity $J$ and topographic wavenumber $\chi$, see Figure 4(c). At each $\chi$, as the topographic height $h_{0}$ (and subsequently $J$ ) increases, the near-bottom dissipation also increases. Away from the topography, however, the behavior of the dissipation profiles with depth depends on $\chi$, as we review next.

For the narrow topography $(\chi=1.12), D\left(E_{K}\right)$ is only significant near the bottom, and sharply drops off with height by $\sim 4$ orders of magnitude, such that it is insignificant in comparison to topographies with $\chi<1$ above $z \sim 0.06$. Unlike the simulations with $\chi<1$, in this topographic regime, KE dissipation at greater $\mathrm{HAB}$ (say, $\mathrm{HAB}>0.1$ ) does not strictly increase with $J$. Rather, $D\left(E_{K}\right)$ is the largest at $J=0.6$ and the lowest at $J=1$, which is the simulation that does not exhibit inertial periodicity in $D\left(E_{K}\right)$.

$D\left(E_{K}\right)$ in the wide topographic regime $(\chi=0.063)$, while smaller than that in the radiative regime at the same $J$, does not decay with $\mathrm{HAB}$ as abruptly as in the simulations with $\chi=1.12$. As first predicted by Nikurashin and Ferrari (2010b), dissipation intensity $D\left(E_{K}\right)$ is higher in the radiative 
regime $(\chi=0.16)$, all else being equal. The dissipation rate for the case with $\chi=0.063, J=0.6$ is about $2-3$ order of magnitude lower than those at higher $J$. It also happens to be the only simulation in this topographic regime that did not exhibit inertial periodicity in $D\left(E_{K}\right)$. However, dissipation rates at $\chi=0.063, J=2,5$, which is the more relevant regime for the wide topography, are equal or greater than those at $\chi=0.16, J=0.6$.

In the following sections, we investigate the connection between the inertial periodicity in $\mathrm{KE}$ dissipation and the generation of and the non-linear interactions between internal waves. We further assess the role of hydraulic control and the difference in the effects of higher topographic height across the topographic wavenumber regimes.

\section{Frequency Content of the Velocity Field}

\section{a. Near-inertial wave field}

In order to trace the origins of the inertial modulation of $D\left(E_{K}\right)$, we first investigate whether propagating near-inertial waves are generated in these simulations. The top panel in Figure 5 shows the temporal evolution of horizontally averaged normalized perturbation velocity $u / U$ for simulations in different regimes at the fixed value of $J=0.6$ to compare across $\chi$ : (a) wide $(\chi=0.063)$, (b) radiative $(\chi=0.16)$, and (c) narrow $(\chi=1.12)$ topographies. Note that in the presence of a mean flow, purely inertial waves can either have flat phase lines, which the horizontal average captures, or propagate at an angle (see §5a and appendix), with a horizontal wavenumber that is a multiple of $k$, and which the horizontal average filters out. Nonetheless, these plots are sufficient to demonstrate our point qualitatively. In order to capture all waves, we horizontally average the frequency spectra and bispectra (discussed in the next subsection) only after computing the spectra and bispectra for each vertical "cast" at locations across the ridge. We first present the 
results for $J=0.6$ as it corresponds to the typical topographic height parameter considered for the radiative regime in previous studies (e.g., Nikurashin and Ferrari 2010a; Klymak 2018).

For $\chi>|f| / N$ (radiative and narrow topographies) and $J>0.6$, we find qualitatively similar results at different $J$, while the differences at greater $J$ for $\chi=0.063$ are discussed below. We observe radiating near-inertial waves, even when the topographic wavenumber is not within the lee wave radiative regime. After the initial spin-up, which we recall to have defined as the first $4 t_{I}$, internal waves develop with a periodicity equal to the inertial period. The dominant signal of these inertial oscillations is evident in the peak of the perturbation velocity spectra at the frequency $\omega=f$, both near and away from the bottom (see horizontally averaged frequency spectra for $u / U$ plotted with depth in the bottom panels of Fig. 5).

It is important to note that in the case of $\chi=0.063$ (wide topography), the magnitude of the inertial oscillations increases with the topographic height, comparing Fig. 5(a) for $J=0.6$ with Fig. 7(a,d) for $J=2,5$. At $J=0.6$, the inertial motions are relatively weak for $\chi=0.063$ compared $\chi=0.16,1.12$. We also do not observe inertial modulations of $D\left(E_{K}\right)$ for this simulation, and $D\left(E_{K}\right)$ is substantially lower than in the radiative regime. Comparing across $\chi$ at a fixed $J$, one may conclude that flows over wide topography contribute insignificantly to the inertial wave generation and KE dissipation. However, recall that in the ocean, wider hills tend to have greater heights, e.g. $J>3$ in the Southern Ocean for the wide topographies estimated by Klymak (2018). We find that in our wide topography simulations with $J=2,5$, inertial oscillations are an order of magnitude greater than at $J=0.6$ and that $D\left(E_{K}\right)$ exhibits inertial periodicity. Hence, inertial oscillations may indeed play an important role in the wide topographic regime as well. 


\section{b. Rotary spectra and bispectra}

In order to understand the generation mechanisms of the near-inertial signal, we compute rotary spectra for the complex velocity $u+i v$ in frequency space over the last $4 t_{I}$, which we then horizontally average at $\mathrm{HAB}=0.1$. The top panel of Figure 6 shows the results for (a) wide $(\chi=0.063),(b)$ radiative $(\chi=0.16)$, and $(c)$ narrow $(\chi=1.12)$ topographies in the linear regime $(J=0.6)$. At the inertial frequency, especially for the radiative and narrow topographies, rotary spectra have a strong counterclockwise component, consistent with the direction of rotation of bottom-radiated inertial waves in the Southern Hemisphere. The flow is accelerated when it goes over the topography, as shown in Fig. 1, and is deflected to the left by the Coriolis force in the Southern Hemisphere, resulting in a counterclockwise-rotating wave. Same dominant peak at the counterclockwise-polarized near-inertial frequency was observed in the ADCP velocities, e.g. in the western Scotia Sea by Brearley et al. (2013); Cusack et al. (2020) and over the the Kerguelen Plateau by Waterman et al. (2014).

There are additional relative peaks in rotary spectra at the super-harmonics (i.e., waves with frequencies that are multiples of $f$ ) in all topographic regimes, but these peaks are especially prominent in the radiative topography (cf. Fig. 6(b)). In order to investigate whether the peaks are the result of energy transfer to higher frequencies due to non-linear resonant interactions, we compute bispectra (McComas and Briscoe 1980) corresponding to the same complex velocities as Fig. 6(a-c). To do so, we use the pycurrents package that is part of the UHDAS software (Firing et al. 2012). Non-linear interactions between two internal waves with frequencies and wavevectors $\left(\omega_{1}, \mathbf{k}_{1}\right)$ and $\left(\omega_{2}, \mathbf{k}_{2}\right)$ produce a third wave with $\left(\omega_{3}=-\left(\omega_{1}+\omega_{2}\right), \mathbf{k}_{3}=-\left(\mathbf{k}_{1}+\mathbf{k}_{2}\right)\right)$. When these waves satisfy the resonance conditions $\left(\omega_{i}\left(\mathbf{k}_{i}\right), i=1,2,3\right.$, all follow the internal wave dispersion relation), bispectrum is non-zero at those frequencies. 
The rotary bispectra for three different topographic regimes computed at $\mathrm{HAB}=0.1$ are shown in the lower row of Figure 6. The bispectrum of a single process (here $u+i v$ ) is symmetric across the $\left(\omega_{1}=\omega_{2}\right)$ axis (Neshyba and Sobey 1975; Chou 2013) with colors indicating the strength of the non-linear resonant wave-wave interactions. Bispectral energy of the non-radiative domains (Fig. 6(d,f)) is significantly lower than that of the radiative topography (Fig. 6(e)). In the radiative regime, resonant interactions are an important mechanism for energy transfer to higher frequencies. These interactions are particularly strong between a wave at inertial frequency and the super-harmonics, especially the counterclockwise-rotating $\omega_{1} / f=\omega_{2} / f=+1$ and clockwiserotating superharmonics $\left(\omega_{1}, \omega_{2}\right) \sim-f$, consistent with the rotary spectra (cf. Fig. 6(b)).

On the other hand, the non-linear interactions are only weakly resonant in the non-radiative regimes. In the narrow topographic regime (Fig. 6(f)), the primary interaction is between counterclockwise-rotating inertial waves, which is the strongest rotary spectrum signal (cf. Fig. 6(c)).

For the wide topography ( $\chi=0.063$ ), the rotary spectrum signal is small at $J=0.6$ (Fig. 6(d)), but increases with $J$ (Fig. 7(c,f)). At $J=0.6$, the zero-frequency flow has greater spectral energy than $\omega=f$ signals in this simulation (Fig. 6(a)). As the topographic height increases $(J=2,5)$, the nearinertial wave is greater in magnitude (Fig. 7(a,d)). As a result, we see stronger resonant interactions along $\omega_{1} / f=\omega_{2} / f= \pm 1$. Yet, the rotary bispectrum is still at least an order of magnitude lower even at $J=5$ (Fig. 7(f)) compared with the radiative linear regime $(\chi=0.16, J=0.6$, Fig. 6(e)), and we do not observe significant bispectrum signal at the super-harmonic frequencies, unlike the radiative regime. This indicates that the origin of the inertial oscillations in the wide topography case may be of non-resonant nature.

Indeed, the waves resulting from resonant interactions are freely propagating (Frajka-Williams et al. 2014) and carry energy into the ocean interior leading to instabilities and turbulence (Garrett 
and Kunze 2007). However, Grisouard and Thomas (2015) have shown that the non-linear interaction of non-resonant (or possibly very weakly resonant) forced waves could also generate motions that help energy propagate into the interior. The results in this section indicate that there is a strong correlation between the bottom-radiated near-inertial motions and dissipation rates, even over the non-radiating topographies. In the following section, we investigate the non-linear interactions, both resonant and non-, or weakly, resonant, relating them back to the inertial modulations of KE dissipation presented in $\S 3$.

\section{Non-linear forcings}

\section{a. Methodology}

In this section, we introduce complex modulation (CD) filtering, which has previously been applied to internal waves (Mercier et al. 2008; Grisouard and Thomas 2015), to isolate the spatial structure of the flow at a given frequency and study the interactions that give rise to motions at these frequencies.

While the energy spectra in the simulations have signal over the whole frequency continuum, there are noticeable relative peaks at distinct harmonic frequencies (e.g., Fig. 6(a-c)). Following the previous CD filtering studies, and because we are mostly interested in a somewhat qualitative description of the flow, we can reasonably approximate the across-ridge flow as a sum of discrete harmonics of $f$, namely,

$$
u(x, z, t) \approx \sum_{n=-\infty}^{\infty} U_{n f}(x, z) e^{i n f t}
$$

where $U_{n f}$ is the spectral component at frequency $n f$, defined as

$$
U_{n f}(x, z)=\frac{1}{T} \int_{t_{0}}^{t_{0}+T} u(x, z) e^{-i n f t} d t
$$


The contributions from intermediate frequencies are also non-zero; however, the goal here is to isolate and study the motions at a few low-frequency harmonics. It is important to note that from the hydrostatic dispersion relation (see derivation in Appendix B), a freely propagating inertial wave can travel along two characteristics with slopes $\left(\alpha_{1}, \alpha_{2}\right)$ corresponding to the two horizontal wavenumbers $\left(k_{1}, k_{2}\right)$ that are roots of the hydrostatic dispersion relation (cf. Eqn. (B1)). The two slopes of the wave characteristics are

$$
\alpha_{1}=0, \quad \alpha_{2}=\sqrt{\frac{U k_{2}\left(U k_{2}+2 f\right)}{N^{2}}} .
$$

$U_{n f}$ is complex, and because $u$ is real, $U_{-n f}$ equals the complex conjugate of $U_{n f}$. Substituting Eqn. (8) into the $x$-momentum equation, we can obtain the non-linear terms that force motions at frequency $n f$, namely,

$$
\Lambda_{n f}=\frac{1}{T} \int_{t_{0}}^{t_{0}+T}-\left(u u_{x}+w u_{z}\right) e^{-i n f t} d t=\sum_{j=0}^{\infty} \Lambda_{n f}^{(j)} .
$$

Here, $t_{0}$ is a given initial time, large enough for the dynamics to have become reasonably stationary, and which we take as $t_{0}=4 t_{I}$ for all simulations except $(\chi, J)=(0.16,0.6)$ and $(\chi, J)=(1.12,0.4)$, where $t_{0}=8 t_{I}$. We choose $T=4 t_{I}$, multiple of $2 \pi /(n f)$. Finally, $\Lambda_{n f}>0(<0)$ indicates the energy transfer that enhances (diminishes) the amplitude of $\omega=n f$ oscillations.

$\Lambda_{n f}^{(j)}$ represents the triadic non-linear interaction between the wave at frequency $n f$ and two other waves with frequencies $I=(\lfloor n / 2\rfloor-j) f$ and $J=(\lceil n / 2\rceil+j)$, such that $I+J=n f$ and $\lfloor\cdot\rfloor,\lceil\cdot\rceil$ are the floor and ceiling operators, respectively. The non-linear motions that force the $n^{\text {th }}$ harmonic are the sum of the infinite number of triadic interactions with the waves of frequencies that add up to $n f$. For example, for the inertial oscillations $(n=1)$, the non-linear forcing $\Lambda_{f}$ can be due to the interactions between waves with frequencies $(0,-f, f)$, i.e., $\Lambda_{f}^{(0)} ;(f, f,-2 f)$, i.e., $\Lambda_{f}^{(1)}$, and so on. 
We now focus on each topographic wavenumber regime individually and compare the results across the topographic heights. In addition to quantifying the non-linear interactions, we also identify regions where the flow is unstable by computing the local instantaneous Richardson

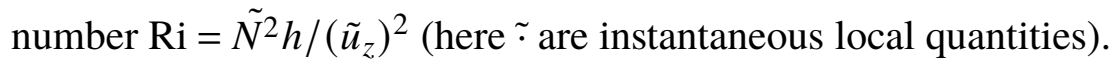

\section{b. Radiative topography $(|f| / N<\chi<1)$}

\section{1) NON-LiNEAR WAVE-WAVE INTERACTIONS}

Figure 8 shows the CD-filtered and normalized velocities $U_{0} / U, U_{f} / U$ and $U_{2 f} / U$ in the top panel, and the non-linear forcing for the inertial motion $\left(U_{f}\right)$ broken down into components across the bottom panel for the simulations with $\chi=0.16, J=0.6$. The zero-frequency flow $U_{0} / U$ (Fig. 8(a)) exhibits waves that propagate away from the topography. The vertical wavelength of these waves is $\lambda_{z} \sim 2 \pi U / N$, corresponding to the vertical wavelength of the lee waves from linear theory. We also observe freely propagating inertial waves, which we identify from their slopes $\alpha_{1}, \alpha_{2}$ defined in Eq. (10) and vertical wavenumber $m_{I O}=k / \alpha_{2}$, with $\alpha_{2}$ computed from Eq. (B3), taking $k_{2}=k=2 \pi / L_{x}$, the topographic wavenumber. These freely propagating inertial waves are expected in the radiative regime, as strong resonant wave-wave interactions appear in the bispectra (cf. Fig. 6(e)).

There are substantial contributions to the non-linear forcing $\Lambda_{f}$ (Fig. 8(d)) from both the interactions between the lee waves $U_{0}$ and inertial oscillations $U_{f}\left(\Lambda_{f}^{(0)}\right.$, Fig. 8(e)), and between $U_{f}$ and $U_{2 f}\left(\Lambda_{f}^{(1)}\right.$, Fig. 8(f)). While $\Lambda_{f} \approx \Lambda_{f}^{(0)}+\Lambda_{f}^{(1)}$, the comparison between Figs. 8(d,g) illustrates that there are non-zero contributions from the interactions among the higher harmonics. Notably, there are especially strong wave-wave interactions that transfer energy to the inertial frequency $\left(\Lambda_{f}>0\right)$ downstream of the topography. 
Same patterns appear in the simulations with taller topography $(J=1,2): U_{0} / U$ corresponds to propagating lee waves (Fig. 9(a,d)) and $U_{f} / U$ to the inertial waves (Fig. 9(b,e)). The inertial oscillations increase in magnitude with $J$, and the non-linear interactions between the lee waves and inertial oscillations are stronger (Fig. 9(c,f)), in particular downstream directly above the topography where the energy is transferred to the inertial frequency, as shown by verticallyaveraged $\Lambda_{f}^{(0)}$ over $z \in[0,0.5]$ being positive. On the upstream side, $\Lambda_{f}^{(0)}<0$, i.e. the inertial waves actually lose energy. However, the horizontally-averaged non-linear forcing is positive in the region above the topography $(z<0.1$, Fig. $9($,h) $)$ and positive downstream, suggesting that overall inertial motions are generated there. We will now investigate this region further through the lens of hydraulic control.

\section{2) Hydraulic CONTROL}

Figure 10 shows streamlines with colors representing $u / U$ across the top panel and Ri across the bottom panel plotted at $t=6.5 t_{I}$ for different $J$ at $\chi=0.16$. The flow is faster downstream of the topography for all $J$, such that it is asymmetric between the up- and downstream sides. The asymmetry is more apparent at larger $J$ : close to the topography, as a layer of fluid goes over the obstacle, it accelerates and narrows, exhibiting characteristics of hydraulic control (Klymak et al. 2010; Winters and Armi 2012).

When the flow accelerates over the topography, it creates a horizontal gradient $\partial_{x} u>0$ (e.g., $\partial U_{0} / \partial x>0$, cf. Figs. 8(a) and 9(a,d)). Because we are in the southern hemisphere, the accelerated flow is also turned by the Coriolis force counterclockwise, such that $U_{f}<0$ directly above the topography (cf. Figs. 8(b) and 9(b,e)). This flow dynamics results in the positive energy transfer to the inertial frequency downstream of the topography, i.e. the non-linear term $-U_{f}\left(\partial U_{0} / \partial x\right)>$ 0 . As $J$ increases, the accelerated layer becomes faster and narrower, which leads to larger 
horizontal velocity gradient $u_{x}$ and stronger $U_{f}$, as the Coriolis force is proportional to the velocity. Subsequently, the $-u u_{x}$ component of the non-linear term $\Lambda_{f}$ becomes larger, further reinforcing the inertial oscillations. The specific dynamics of $\Lambda_{f}>0(<0)$ downstream (upstream) of the topography need to be investigated in a follow-up study via careful analysis of the energetics, in particular energy exchange between the time-mean and fluctuating kinetic energy reservoirs. However, here we observe that overall energy is transferred to the inertial field near topography through non-linear interactions, which increase with $J$. Similarly, energy transfer to the fluctuating (commonly referred to as "eddy") KE from the mean KE downstream of topography was observed in the non-rotating simulations by Jagannathan et al. (2020).

Moreover, Nikurashin and Ferrari (2010b) noted that inertial oscillations create strong vertical shear, which we also find in our simulations, shown by the regions of critical Richardson number $(\mathrm{Ri}<0.25)$ in Figs. 10(d-f). As $J$ increases and the effects of hydraulic control strengthen, the unstable region extends even further above the topography, and a region of convective overturning $(\mathrm{Ri}<0)$, which we showed in Fig. 1, appears and expands. According to Nikurashin and Ferrari (2010b), this vertical shear further enhances breaking of the lee waves. We similarly find that near the topography, KE dissipation increases with $J$ (Fig. 4(b,e,f)), as the flow downstream of the topography becomes more turbulent and stronger inertial waves are generated, leading to localized breaking. This increased local breaking also results in the generation of stronger inertial motions, in part through non-linear wave-wave interactions in the downstream region where $\Lambda_{f}>0$. Inertial waves in turn create stronger shear leading to enhanced breaking away from the topography. Subsequently, inertial periodicity in $D\left(E_{K}\right)$ develops at an earlier time for the simulations at $J=1,2$ and propagates further into the interior (Figs. 3(e,h)) compared with the $J=0.6$ case.

It is important to note for all simulations in this radiative regime, the lee waves are of equal magnitude to the inertial waves, allowing for strong non-linear interactions to occur. In the 
remainder of this section, we apply the same analysis to the simulations in the non-radiative regimes to whether both inertial and lee waves are present and how their interactions contribute to the inertial modulation of KE dissipation.

c. Narrow topography $(\chi=1.12)$

Figure 11 shows the CD-filtered and normalized inertial harmonic $U_{f} / U$ in the top row and the non-linear forcing $\Lambda_{f}$ in the middle row for $\chi=1.12, J=0.4,0.6$ and 1. At this $\chi$, the non-linear interactions are primarily confined to the region directly above the bottom. For all $J$, we observe propagating inertial waves (Fig. 11(a-c)) with the slope corresponding to $\alpha_{1}=0$; however, the vertical distribution varies with $J$.

At $J=1$, both the zero-frequency flow and the inertial oscillations are the strongest directly above the topography in the region where the non-linear forcing is strong, indicated by the frequency spectrum of $u / U$ (Fig. 11(i)). However, the magnitude of $U_{f}$ decreases sharply with $z$, also shown by the drop in $\log _{10} \phi_{u}^{t}$ at $\omega / f=1$. At lower $J$, stronger inertial oscillations propagate further away from the topography, especially at $J=0.6$, and there is no substantial change in the spectrum at $\omega / f=1$ with $z$ (Fig. $11(\mathrm{~g}, \mathrm{~h})$ ). At all $J, U_{0}$ is substantially weaker than the inertial motions away from the topography. In the simulation with $J=1$, it especially decreases with HAB and is much weaker than for the simulations with $J=0.4,0.6$ away from the topography, which do not exhibit such substantial change with height.

In Figure 12(a-c), we plot snapshots of the streamlines at $t=6.5 t_{I}$, including the LOTS, for the simulations with $\chi=1.12, J=0.4,0.6,1$, respectively. As $J$ increases, the flow becomes progressively more blocked and the LOTS more separated from the topography. At $J=1$, the LOTS is almost horizontal and also develops undulations, resulting in substantial modifications to the effective topography. 
The results from these simulations suggest that the inertial periodicity of $D\left(E_{K}\right)$ (cf. Fig. 3(c,f,i)) only occurs in cases where both the inertial motions $\left(U_{f}\right)$ and at least weak zero-frequency flow $\left(U_{0}\right)$ are present. Indeed, the greater topographic height does lead to stronger $U_{0}$ and $U_{f}$ directly above the topography, where the stronger Coriolis force results from faster accelerated flow. The non-linear interactions and turbulent motions are also stronger in this region at higher $J$, as shown by $\Lambda_{f}$ (cf. bottom panels of Fig. 11) and KE dissipation increases with $J$ near the topography (Fig. 4). However, as the effective topography flattens and the flow becomes more blocked, such motions are confined to the region near topography. At $J=1, U_{0}$ and subsequently the non-linear interactions between $U_{0}$ and $U_{f}$ are reduced, and $\mathrm{KE}$ dissipation away from the topography is smaller compared with the cases with stronger zero-frequency flow $(J=0.4,0.6)$, for which we observe inertial modulation of $D\left(E_{K}\right)$. However, even in those simulations $(J=0.4,0.6)$, the nonlinear interactions and the KE dissipation rates in the interior are at least $1-2$ orders of magnitude lower than the simulations with radiative or wide and tall topographies (i.e., $\chi=0.16, J=0.6,1,2$ and $\chi=0.063, J=2,5)$.

\section{d. Wide topography $(\chi=0.063)$}

The CD-filtered inertial harmonic $U_{f}$ and the non-linear forcing term $\Lambda_{f}$ are shown for $\chi=$ $0.063, J=(0.6,2,5)$ in Figure 13. In these simulations, $U_{2 f}$ and, subsequently, $\Lambda_{f}^{(1)}$ are negligible and hence not shown here. Hence, the non-linearly forced motions at the inertial frequency are primarily driven by the interactions between $U_{0}$ and $U_{f}$, which differs from the radiative regime, where the resonant interactions with the second and higher harmonics play an important role.

Unlike the narrow topographic regime, here we find strong time-mean flow $U_{0}$ at all values of $J$ (Figs.13(a,d,g)) as shown by the rotary spectra. The phase lines of $U_{f}$ are horizontal (i.e., $\alpha_{1}=0$ ) only directly above the topography, and are inclined above. The slope of the characteristics, 
however, does not exactly align with the slope of the freely propagating inertial motions (i.e., $\alpha_{2}$ ). Following Grisouard and Thomas (2015), this mismatch can be attributed to the non-resonant, rather than resonant, non-linear interactions. This is consistent with our earlier observation that rotary bispectra were much weaker at $\chi=0.063$ compared with $\chi=0.16$ (cf. Figs. 6, 7).

Comparing across Figs. 13(a,c,e), $\left|U_{f}\right|$ is an order of magnitude greater for the non-linear topographies $(J=2,5)$ than the linear topographies $(J=0.6)$. This is in contrast with the narrow regime, where greater non-linearity coincided with less near-inertial signal. Here, the non-linear forcing and, subsequently, the non-linear interactions between $U_{0}$ and $U_{f}\left(\Lambda_{f} \approx \Lambda_{f}^{(0)}\right)$ are also substantially stronger at larger $J$ (Figs. 13(b,d,f)). For $J>1$, the flow becomes more turbulent, particularly downstream of the topography as indicated by $\mathrm{Ri}<0.25$ (Fig. 14(e,f)). In these cases, the flow is visibly asymmetric between the up- and the downstream (Fig. 14(b,c)). $\Lambda_{f}$ is large and positive, especially in the regions where the convective overturns and wave breaking occur. For $J=2$ and 5 , the vertical average of $\Lambda_{f}$ over $z \in[0,0.5]$ is predominantly positive downstream, and the horizontal average is predominantly positive directly above the topography (cf. Fig. 13d,f). As such, the hydraulics downstream of the topography coincide with the inertial wave generation and the inertial motions appear to be enhanced through $\Lambda_{f} \approx \Lambda_{f}^{(0)}$, i.e. the interaction with $U_{0}$. Unlike the radiative linear simulation $(\chi=0.16, J=0.6)$ and the narrow topography simulations $(\chi=1.12)$ where the counterclockwise inertial oscillations are dominant, we find clockwise and counterclockwise inertial motions of approximately similar magnitude for wide topographies (cf. Fig. 7). It further suggests that over such wide topographies, another mechanism, in addition to the Coriolis force turning the accelerated flow counterclockwise, may be responsible for generating these inertial motions.

Interestingly, at $\chi=0.063, J=5$, we also find that the LOTS detaches from the topography and develops undulations. In this case, mechanisms analogous to the evanescent masking and 
evanescent undulation (Mayer and Fringer 2020) may actually increase the lee wave drag in this case due to the narrower effective topography and greater effective $\chi$. Thus, the effects of an increase in the topographic height on wave generation and radiation can differ between the wide and narrow topographic regimes, even though linear theory only predicts the generation of evanescent lee waves in both.

In the presence of the instabilities that arise in the region of hydraulic control, at $J=2$ and 5 we find stronger non-linear interactions between $U_{0}$ and $U_{f}$ (i.e. $\left.\Lambda_{f}^{(0)}\right)$ and inertial modulation of $D\left(E_{K}\right)$, unlike the simulation with $J=0.6$, where $\Lambda_{f}^{(0)}$ is much weaker and there is no inertial periodicity in $D\left(E_{K}\right)$. The stronger inertial oscillations create vertical shear away from the topography, facilitating wave breaking not only near the topography localized in the region of hydraulic jump, but also further away from the topography. In our simulations (cf. Fig. 4), the $D\left(E_{K}\right)$ for tall wide topography ( $\chi=0.063, J=2$ and 5) does not abruptly decay with height above the bottom. In magnitude, it is comparable or can exceed that of the linear radiative regime $(\chi=0.16, J=0.6)$, which is important for the ocean given its red topographic spectrum.

\section{Connecting the topographic width and height regimes}

The results of this study most importantly highlight the dependence of the flow dynamics on both the topographic width (i.e., $\chi$ ) and non-linearity of the flow (i.e., $J$ ). Previous works of the flow above rough topography have often focused on comparing between radiative and non-radiative regimes (i.e., across $\chi$ ) or between linear and non-linear regimes (i.e., across $J$ ). However, we find that the internal wave generation and dissipation rates depend on the combination of $\chi$ and $J$, rather than either of the parameters alone. Indeed, inertial modulations of the KE dissipation that facilitate further dissipation in the interior (cf. Fig. 3) only do not occur in the simulations with either narrow and tall hills or wide and short hills. These results suggest that another parameter, such as the 
slope of the hill $\xi \sim J \chi$, could be the more relevant parameter. While acknowledging that the particular details of such dependency need to be addressed in further studies either through theory or additional numerical simulations and without claiming that $\xi$ is this parameter, we summarize our general observations below.

In our simulations, we find that on one hand, too steep a slope may lead to substantial blocking of the flow. On the other hand, too shallow a slope may prevent the development of a hydraulic jump and subsequent generation of instabilities downstream of the topography. However, in some intermediate range of topographic slope, generation of inertial motions, non-linear wave interactions, and subsequently dissipation rates could be enhanced. In our simulations, this intermediate range of $\xi$ corresponds to narrow and short, radiative, and wide and tall topographies.

In agreement with previous work (Nikurashin and Ferrari 2010b,a), we find that the inertial periodicity in dissipation over the radiative topographies corresponds to the presence of both lee waves and inertial oscillations. However, we find that if including non-linear effects, the inertial waves can not only be freely propagating, but also non-linearly forced. This extends the regime where strong dissipation occurs to non-radiative topographies. Moreover, the horizontally-averaged dissipation rates are higher in the simulations where the non-linear interactions (resonant, weakly resonant, or non-resonant) between the time-mean flow and inertial oscillations are strong.

In the case of narrow topography, while inertial oscillations develop and can radiate away from the topography, the mean flow is very weak. The narrow hills have comparatively much steeper slopes at a fixed $J$. As discussed by Mayer and Fringer (2020), it leads to a more blocked flow, such that the effective topography felt by the flow becomes substantially different from the bottom topography. Thus, wave drag is reduced through both evanescent masking and undulations. As a result, we observe only weak inertial modulations at smaller hill heights $(J=0.4$ and 0.6 corresponding to 
$\xi=0.15,0.21)$, respectively, and no signature of them for $(J, \xi)=(1,0.35)$. Accordingly, the dissipation rates in the interior above the narrow hills are also small.

In the case of wide topography, inertial oscillations only develop above hills with sufficient non-linearity $(J>2$ in simulations, corresponding to $\xi>0.080)$, where hydraulic control of the flow facilitates their generation and stronger non-linear interactions between the zero-frequency and inertial waves are present. Such inertial motions are absent above the hill with $J=0.6$, which corresponds to a shallower slope $\xi=0.012$, resulting in smaller dissipation rates. Notably, Nikurashin and Ferrari (2010b) found the inertial oscillations were also absent in some of their simulations in the radiative regime, for which the slope was $\xi<0.029$. In these simulations, dissipation was similarly reduced compared to the ones where the inertial oscillations were present, further hinting at $\xi$, rather than $\chi$ or $J$ alone, could be the relevant parameter. In our simulations, dissipation rates above wide and taller hills $(\chi=0.063, J>2)$, where the inertial motions do develop, are comparable or can exceed that of the linear radiative regime $(\chi=0.16, J=0.6$, $\xi=0.031)$. Taking into consideration that the typical values of $J$ in the ocean are $\sim 3$ for wide and $\sim 0.6$ for radiative topographies (Klymak 2018), this result implies that the wide topographies could have a significant contribution to the KE dissipation rates both near the topography and in the ocean interior.

\section{Discussion and summary}

In this study, we extended the idealized simulations of Nikurashin and Ferrari (2010b) to abyssal topographies with wavenumbers outside of the lee wave radiative regime. Specifically, we examine the effects of topographic width and height on KE dissipation rates, and some of our simulations present inertial periodicity in KE dissipation. We draw three main conclusions regarding the vertical distribution of the horizontally averaged dissipation rates: 
1. within the same topographic width regime (i.e., $\chi$ ), near the topography, dissipation rates increase with nonlinearity (i.e., $J$ );

2. at the same $\chi$, away from the topography, dissipation rates are greater for simulations with inertial modulation of dissipation, and

3. while flow over narrow abyssal hills (i.e., $\chi>1$ ) does not yield significant dissipation, the dissipation rates above wide and tall hills (i.e., $\chi<|f| / N, J>1$ ) can be comparable to those above hills within the lee wave radiative regime (i.e., $|f| / N<\chi<1$ ).

The flow has to accelerate to go over the hill, and is deflected by the Coriolis force (to the left in the Southern Hemisphere), and a counterclockwise inertial wave is generated. Such inertial waves with upward-propagating phase close to the rough bottom topography have been previously observed (e.g., in the Southern Ocean by Waterman et al. (2014) and in the eastern Pacific by Alford (2010)), but have been attributed to the propagation of the surface wind-driven near-inertial energy to great depths. As the topographic height increases, there is greater asymmetry in the flow between the upstream and downstream of the abyssal hill akin to a sub-to-supercritical hydraulic transition. It results in a flow, more prone to shear and even convective instabilities, and loss of geostrophic balance, such that non-linear motions become important near the topography. The region where these instabilities occur is co-located with the region of near-inertial wave generation for taller topographies.

Inertial waves create vertical shear, which then leads to wave breaking near the topography. This argument may appear circular, as we mentioned earlier that the wave breaking leads to the inertial wave radiation above the topography. However, as discussed by Nikurashin and Ferrari (2010b), it is more akin to an initial value problem, where the generation of inertial waves is at first facilitated by the instabilities near the topography, and then the inertial waves in turn facilitate 
further wave breaking. As stronger inertial waves are generated above taller hills (greater $J$ ), $\mathrm{KE}$ dissipation rates also increase with $J$ directly above the topography. Vertical shear was found to play an important role in the energy transfer rates from the Southern Ocean observations by Cusack et al. (2020). The authors further found substantial contributions from both vertical stresses and buoyancy fluxes to the vertical energy transfer from the internal wave field to the geostrophic flow near the topography, contrary to the linear theory. Motivated by these observations, it would be illuminating to follow up with a full analysis of the energy budget focused on the wave-mean flow energy transfer rates using different topographic regimes.

For all simulations in the radiative regime, we observe inertial periodicity in KE dissipation. In this regime, the steady lee waves and inertial waves are of similar magnitude, and their resonant nonlinear interactions enhance breaking and dissipation, as was previously explained by Nikurashin and Ferrari (2010b). In contrast, above the narrow hills, effective topography is nearly horizontal due to blocking and subsequent evanescent masking (Mayer and Fringer 2020). Non-linear effects are only present directly above the topography and the dissipation rates are small. Above a short and wide topography, the generated inertial waves are weak, and the dissipation rates are also small. However, for a taller topographic feature, instabilities arising in the vicinity of hydraulically controlled flow help generate stronger inertial waves, leading to stronger non-linear interactions and wave breaking. Subsequently, in our simulations flow above wide and tall abyssal hills exhibits inertial periodicity in KE dissipation and increased dissipation rates away from topography.

The contributions to the upward radiated energy flux from abyssal topographies with wavenumbers $k<|f| / U$ have been typically disregarded by other authors because according to linear theory, no freely propagating internal waves are radiated in this regime, and they have no lee waves to interact with. However, Bell's linear theory is only applicable when $J$ is small (Bell 1975). Yet, in the ocean, wide (small $k$ ) topographies tend to be higher (greater $h_{0}$ and subsequently $J$ ) in 
comparison with the radiative topographies. As such, based on the calculations of $J$ for various wavenumber topographies by Klymak (2018), it may be more relevant to compare (1) radiative topographies with $J<1$ to (2) tall and wide non-radiative topographies with $\chi<|f| / N, J>1$. Our simulations show that the kinetic energy dissipation rates resulting from the geostrophic flow over such topographies is comparable for the regimes in the ocean interior. Furthermore, the dissipation rates directly above the topography in regime (2) exceed those in regime (1) due to stronger effects from the hydraulic control. These conclusions are consistent with the simulations with realistic multichromatic topography by Klymak (2018). Furthermore, those simulations showed that the scales couple, as simulations with the full topographic range (radiative and large scales) had dissipation rates greater than the sum of the simulations with radiative and large scale topographies alone. As a result, contrary to the linear lee wave theory, abyssal topographies in the non-radiative regime may have an important contribution to energy dissipation and ocean mixing because of the non-linear interactions.

The wide $(\chi<|f| / N)$ regime does not only apply when the flow goes over wide topographic obstacles, but also when the velocity $U$ of the flow over an obstacle, whose wavenumber was previously within the radiative regime, decreases. A decrease in $U$ corresponds to an increase in non-linearity $J$, and such flows could play an important role in local dissipation and mixing, yet they would be excluded in the linear lee wave drag parameterizations. The sensitivity of the flow specifically to $U$-driven changes in $\chi$ in the current formulation will be the subject of future studies, but the effects of increased background velocity on the dissipation and form drag are discussed in Klymak et al. (submitted).

Bell's linear theory provides a formula for computing the energy flux generated by the lee waves (Bell 1975; Nikurashin and Vallis 2011). This estimate can be then used to compute dissipation rates (and furthermore mixing rates) in the abyssal ocean by connecting this lee wave energy 
radiation rate to the dissipation rates integrated over some depth (Gregg et al. 2003; Polzin et al. 2014). However, the direct microstructure measurements of the dissipation rates in the Southern Ocean have been $2-10$ times smaller than the rates predicted from lee wave generation and the finescale parametrization (e.g. Sheen et al. 2013; Waterman et al. 2014; Cusack et al. 2017; Ijichi and Hibiya 2017). Many explanations have been proposed for this discrepancy, as discussed in detail in Kunze and Lien (2019).

According to the mechanism proposed in this paper, the non-linear dynamics play an important role due to the near-topography hydraulic control, enhancing the dissipation rates very close to the topography. This enhanced dissipation, in turn, would decrease the flow velocity above the region of hydraulic control, and as a result, the energy radiation rate would be in fact less than the rate predicted by linear theory using the near-bottom flow speed. The microstructure measurements from the Southern Ocean Finestructure and the Diapycnal and Isopycnal Mixing Experiment in the Southern Ocean campaigns presented in Waterman et al. (2014) support this explanation. As shown in their Figure 14, the dissipation rates are especially elevated directly above the topography (up to $\approx 100-250 \mathrm{~m}$ ), and the flow speed decreases with height in this region. They also find near-inertial intrinsic wave frequency and greater counterclockwise than clockwise polarization in the bottom-most $500 \mathrm{~m}$, consistent with the strong bottom-generated inertial oscillations that we find in this study.

In this study we consider a 1D topography, which does not allow the flow to go around the obstacle. In addition, the 1D nature of the forcing also prevents the generation of along-wall structures (Dewar and Hogg 2010; Venaille 2020, and references therein), which might further modify the dynamics. Both of these simplifications enhance the effects of forced vertical motion and cross-ridge hydraulic control. Reduced horizontal velocities and dissipation rates have been recorded in studies implementing 2D anisotropic topography (Nikurashin et al. 2014; Trossman 
et al. 2015). Accounting for these effect should be the subject of future studies with a focus on comparison of the energy dynamics among the topographic regimes. In particular, it will be beneficial to compute the energy exchange rates between mean and turbulent KE reservoirs to assess what portion of the bottom-radiated wave energy is reabsorbed back into the balanced mean flow (Kunze and Lien 2019). Additionally, this process study of the mechanism for local internal wave radiation and dissipation does not include the effects of remote dissipation of bottom-radiated waves propagating over a long distance (Zheng and Nikurashin 2019). Nevertheless, the results from the current study can provide insights for isolated wide and long ridges, where the flow is forced over the topography and bottom-generated near-inertial oscillations have been observed (e.g., Liang and Thurnherr 2012).

Acknowledgments. We acknowledge the support of the Natural Sciences and Engineering Research Council of Canada (NSERC) [RGPIN-2015-03684]. We are also grateful for fruitful discussions with Jody Klymak.

Data availability statement. The data generated for these simulations exceeds 5TB and cannot be easily distributed. However, we provided all Nek5000 code necessary to run the simulations presented in this paper and all Python code for used for post-processing at https://github. com/bzemskova/bottom_topography_flow.

\section{APPENDIX A}

\section{Effects of stratification restoration}

In order to avoid initial spin-up effects, Nikurashin and Ferrari (2010b) include a term to restore the stratification to the background state. Stratification can be substantially eroded in the region directly above the topography due to turbulent motions, which would alter the wave radiation 
dynamics. While we do not include such relaxation in our simulations presented in the main text to avoid unphysical forcing, we validate this choice here by comparing the results from simulations in $\chi=0.16, J=2$ (lee wave radiative, non-linear) regime both with and without stratification restoration.

For the validation simulation, we add a restoration term to the buoyancy equation of the form $-\tau(b(x, y, z, t)-B(z))$. For the first $2 t_{I}$, we set $1 / \tau=t_{I} / 8$, such that any initial transient effects of the flow adjusting to the bottom topography are smoothed. We then increase the restoration time period to $1 / \tau=3 t_{I}$, such that the waves and turbulence are minimally affected by this forcing.

Comparison between simulations with and without the restoration term is shown in Figure 7. There are quantitative differences between the results. Mainly, without the restoration, the stratification is eroded further above the topography and the kinetic energy dissipation is greater by about a factor of 3 for $z>0.3$. However, the stratification reduction region is confined to approximately $z \leq h_{0}+U / N$, which is the thickness of the bottom layer where buoyancy frequency decays in previous studies of flow over periodic hills (Klymak 2018; Mayer and Fringer 2020). The specific effects of the decayed stratification near the topography on the lee wave drag are discussed in detail in Mayer and Fringer (2020). Here, the results of the simulations with and without buoyancy restoration are qualitatively similar, which suggests that the mechanisms presented in the main text of this paper hold.

\section{APPENDIX B}

\section{Derivation of inertial wave slope}

Assume a perturbation in the form of a two-dimensional plane wave, such that $(u, v, w, b, p)$ are proportional to $e^{i(\omega t+k x+m z)}$, and $\mathbf{k}=(k, 0, m)$ is the wavevector, such that $\alpha=k / m$ is the wave slope. Substituting this plane wave expression into the hydrostatic approximation of Eqns. (??) 
yields dispersion relation

$$
\Omega^{2}=\frac{N^{2} k^{2}+f^{2} m^{2}}{m^{2}},
$$

where $\Omega=\omega+k U$. To solve for the inertial wave, we substitute $\omega=f$ and get two roots for $k$, namely:

$$
k_{1}=0, \quad k_{2}=\frac{2 f U m^{2}}{N^{2}-U^{2} m^{2}} .
$$

Here, $k_{1}$ corresponds to the horizontal inertial wave phase lines, i.e. $\alpha_{1}=0$.

Rearranging the expression for $k_{2}$, we obtain the expression for the vertical wavenumber of the inertial wave:

$$
m_{I O}= \pm N \sqrt{\frac{k_{2}}{U\left(U k_{2}+2 f\right)}},
$$

which yields the second inertial wave slope

$$
\alpha_{2}=\frac{k_{2}}{m_{I O}}= \pm \sqrt{\frac{U k(U k+2 f)}{N^{2}}} .
$$

\section{References}

Alford, M. H., 2010: Sustained, full-water-column observations of internal waves and mixing near mendocino escarpment. Journal of physical oceanography, 40 (12), 2643-2660.

Alford, M. H., J. A. MacKinnon, H. L. Simmons, and J. D. Nash, 2016: Near-inertial internal gravity waves in the ocean. Annual review of marine science, $\mathbf{8}, 95-123$.

Bell, T., 1975: Lee waves in stratified flows with simple harmonic time dependence. Journal of Fluid Mechanics, 67 (4), 705-722.

Brearley, J. A., K. L. Sheen, A. C. Naveira Garabato, D. A. Smeed, and S. Waterman, 2013: Eddy-induced modulation of turbulent dissipation over rough topography in the southern ocean. Journal of physical oceanography, 43 (11), 2288-2308. 
Chou, S., 2013: An empirical investigation of energy transfer from the $\mathrm{m} 2$ tide to $\mathrm{m} 2$ subharmonic wave motions in the kauai channel. M.S. thesis, Department of Oceanography, Univ. of Hawaii at Manoa, Honolulu.

Cusack, J. M., J. A. Brearley, A. C. Naveira Garabato, D. A. Smeed, K. L. Polzin, N. Velzeboer, and C. J. Shakespeare, 2020: Observed eddy-internal wave interactions in the southern ocean. Journal of Physical Oceanography, 50 (10), 3043-3062.

Cusack, J. M., A. C. Naveira Garabato, D. A. Smeed, and J. B. Girton, 2017: Observation of a large lee wave in the drake passage. Journal of Physical Oceanography, 47 (4), 793-810.

De Lavergne, C., G. Madec, J. Le Sommer, A. G. Nurser, and A. C. Naveira Garabato, 2016: The impact of a variable mixing efficiency on the abyssal overturning. Journal of Physical Oceanography, 46 (2), 663-681.

Dewar, W. K., and A. M. C. Hogg, 2010: Topographic inviscid dissipation of balanced flow. Ocean Model., 32 (1-2), 1-13, doi:10.1016/j.ocemod.2009.03.007.

Eliassen, A., 1960: On the transfer of energy in stationary mountain waves. Geophy. Publ., 22, $1-23$

Ezhova, E., C. Cenedese, and L. Brandt, 2018: Dynamics of three-dimensional turbulent wall plumes and implications for estimates of submarine glacier melting. Journal of Physical Oceanography, 48 (9), 1941-1950.

Fabregat Tomàs, A., A. C. Poje, T. M. Özgökmen, and W. K. Dewar, 2016: Dynamics of multiphase turbulent plumes with hybrid buoyancy sources in stratified environments. Physics of Fluids, 28 (9), 095109. 
Firing, E., J. M. Hummon, and T. K. Chereskin, 2012: Improving the quality and accessibility of current profile measurements in the southern ocean. Oceanography.

Fischer, P. F., 1997: An overlapping schwarz method for spectral element solution of the incompressible navier-stokes equations. Journal of Computational Physics, 133 (1), 84-101.

Fischer, P. F., J. W. Lottes, and S. G. Kerkemeier, 2008: Nek5000 web page. URL http://nek5000. mcs.anl.gov.

Frajka-Williams, E., E. Kunze, and J. A. MacKinnon, 2014: Bispectra of internal tides and parametric subharmonic instability. arXiv preprint arXiv:1410.0926.

Garrett, C., and E. Kunze, 2007: Internal tide generation in the deep ocean. Annu. Rev. Fluid Mech., 39, 57-87.

Gayen, B., R. W. Griffiths, and G. O. Hughes, 2014: Stability transitions and turbulence in horizontal convection. Journal of Fluid Mechanics, 751, 698-724.

Goff, J. A., and T. H. Jordan, 1988: Stochastic modeling of seafloor morphology: Inversion of sea beam data for second-order statistics. Journal of Geophysical Research: Solid Earth, 93 (B11), $13589-13608$.

Gregg, M. C., T. B. Sanford, and D. P. Winkel, 2003: Reduced mixing from the breaking of internal waves in equatorial waters. Nature, 422 (6931), 513-515.

Grisouard, N., and L. N. Thomas, 2015: Critical and near-critical reflections of near-inertial waves off the sea surface at ocean fronts. Journal of Fluid Mechanics, 765, 273.

Hu, Q., and Coauthors, 2020: Cascade of internal wave energy catalyzed by eddy-topography interactions in the deep south china sea. Geophysical Research Letters. 
Hughes, G. O., A. M. C. Hogg, and R. W. Griffiths, 2009: Available potential energy and irreversible mixing in the meridional overturning circulation. Journal of Physical Oceanography, 39 (12), $3130-3146$.

Ijichi, T., and T. Hibiya, 2017: Eikonal calculations for energy transfer in the deep-ocean internal wave field near mixing hotspots. Journal of Physical Oceanography, 47 (1), 199-210.

Jagannathan, A., K. B. Winters, and L. Armi, 2020: The effect of a strong density step on blocked stratified flow over topography. Journal of Fluid Mechanics, $\mathbf{8 8 9}$.

Klymak, J. M., 2018: Nonpropagating form drag and turbulence due to stratified flow over largescale abyssal hill topography. Journal of Physical Oceanography, 48 (10), 2383-2395.

Klymak, J. M., D. Balwada, A. Naveira Garabato, and R. Abernathy, submitted: Parameterizing non-propagating form drag over rough bathymetry. Journal of Physica Oceanography.

Klymak, J. M., S. M. Legg, and R. Pinkel, 2010: High-mode stationary waves in stratified flow over large obstacles. Journal of Fluid Mechanics, 644, 321-336.

Kunze, E., and R.-C. Lien, 2019: Energy sinks for lee waves in shear flow. Journal of Physical Oceanography, 49 (11), 2851-2865.

Legg, S., and J. Klymak, 2008: Internal hydraulic jumps and overturning generated by tidal flow over a tall steep ridge. Journal of Physical Oceanography, 38 (9), 1949-1964.

Liang, X., and A. M. Thurnherr, 2012: Eddy-modulated internal waves and mixing on a midocean ridge. Journal of physical oceanography, 42 (7), 1242-1248.

Lvov, Y. V., K. L. Polzin, and N. Yokoyama, 2012: Resonant and near-resonant internal wave interactions. Journal of physical oceanography, 42 (5), 669-691. 
Marshall, J., and K. Speer, 2012: Closure of the meridional overturning circulation through southern ocean upwelling. Nature Geoscience, 5 (3), 171.

Mashayek, A., and W. Peltier, 2012: The'zoo'of secondary instabilities precursory to stratified shear flow transition. part 1 shear aligned convection, pairing, and braid instabilities. Journal of fluid mechanics, 708, 5 .

Mashayek, A., H. Salehipour, D. Bouffard, C. Caulfield, R. Ferrari, M. Nikurashin, W. Peltier, and W. Smyth, 2017: Efficiency of turbulent mixing in the abyssal ocean circulation. Geophysical Research Letters, 44 (12), 6296-6306.

Mayer, F., and O. Fringer, 2017: An unambiguous definition of the froude number for lee waves in the deep ocean. Journal of Fluid Mechanics, 831.

Mayer, F. T., and O. B. Fringer, 2020: Improving nonlinear and nonhydrostatic ocean lee wave drag parameterizations. Journal of Physical Oceanography, 50 (9), 2417-2435.

McComas, C., and M. Briscoe, 1980: Bispectra of internal waves. Journal of Fluid Mechanics, 97 (1), 205-213.

Mercier, M. J., N. B. Garnier, and T. Dauxois, 2008: Reflection and diffraction of internal waves analyzed with the hilbert transform. Physics of Fluids, 20 (8), 086601.

Munk, W., and C. Wunsch, 1998: Abyssal recipes ii: Energetics of tidal and wind mixing. Deep-sea research. Part I, Oceanographic research papers, 45 (12), 1977-2010.

Musgrave, R., J. MacKinnon, R. Pinkel, A. Waterhouse, J. Nash, and S. Kelly, 2017: The influence of subinertial internal tides on near-topographic turbulence at the mendocino ridge: Observations and modeling. Journal of Physical Oceanography, 47 (8), 2139-2154. 
Neshyba, S., and E. Sobey, 1975: Vertical cross coherence and cross bispectra between internal waves measured in a multiple-layered ocean. Journal of Geophysical Research, 80 (9), 1152 1162.

Nikurashin, M., and R. Ferrari, 2010a: Radiation and dissipation of internal waves generated by geostrophic motions impinging on small-scale topography: Application to the southern ocean. Journal of Physical Oceanography, 40 (9), 2025-2042.

Nikurashin, M., and R. Ferrari, 2010b: Radiation and dissipation of internal waves generated by geostrophic motions impinging on small-scale topography: Theory. Journal of Physical Oceanography, 40 (5), 1055-1074.

Nikurashin, M., R. Ferrari, N. Grisouard, and K. Polzin, 2014: The impact of finite-amplitude bottom topography on internal wave generation in the southern ocean. Journal of Physical Oceanography, 44 (11), 2938-2950.

Nikurashin, M., and G. Vallis, 2011: A theory of deep stratification and overturning circulation in the ocean. Journal of Physical Oceanography, 41 (3), 485-502.

Özgökmen, T. M., P. F. Fischer, J. Duan, and T. Iliescu, 2004: Entrainment in bottom gravity currents over complex topography from three-dimensional nonhydrostatic simulations. Geophysical Research Letters, 31 (13).

Polzin, K. L., A. C. N. Garabato, T. N. Huussen, B. M. Sloyan, and S. Waterman, 2014: Finescale parameterizations of turbulent dissipation. Journal of Geophysical Research: Oceans, 119 (2), $1383-1419$. 
Salehipour, H., W. Peltier, and A. Mashayek, 2015: Turbulent diapycnal mixing in stratified shear flows: the influence of prandtl number on mixing efficiency and transition at high reynolds number. Journal of Fluid Mechanics, 773, 178-223.

Shakespeare, C. J., and A. M. Hogg, 2017: The viscous lee wave problem and its implications for ocean modelling. Ocean Modelling, 113, 22-29.

Sheen, K., and Coauthors, 2013: Rates and mechanisms of turbulent dissipation and mixing in the southern ocean: Results from the diapycnal and isopycnal mixing experiment in the southern ocean (dimes). Journal of Geophysical Research: Oceans, 118 (6), 2774-2792.

St. Laurent, L., A. C. Naveira Garabato, J. R. Ledwell, A. M. Thurnherr, J. M. Toole, and A. J. Watson, 2012: Turbulence and diapycnal mixing in drake passage. Journal of Physical Oceanography, 42 (12), 2143-2152.

Talley, L. D., 2013: Closure of the global overturning circulation through the indian, pacific, and southern oceans: Schematics and transports. Oceanography, 26 (1), 80-97.

Trossman, D., S. Waterman, K. Polzin, B. Arbic, S. Garner, A. Naveira-Garabato, and K. Sheen, 2015: Internal lee wave closures: Parameter sensitivity and comparison to observations. Journal of Geophysical Research: Oceans, 120 (12), 7997-8019.

Vanneste, J., 2013: Balance and spontaneous wave generation in geophysical flows. Annual Review of Fluid Mechanics, 45.

Venaille, A., 2020: Quasi-geostrophy against the wall. J. Fluid Mech., 894, R1, doi:10.1017/jfm. 2020.287, URL https://www.cambridge.org/core/product/identifier/S0022112020002876/type/ journal $\left\{\_\right\}$article, 2001.09504. 
Waterman, S., A. C. Naveira Garabato, and K. L. Polzin, 2013: Internal waves and turbulence in the antarctic circumpolar current. Journal of Physical Oceanography, 43 (2), 259-282.

Waterman, S., K. L. Polzin, A. C. Naveira Garabato, K. L. Sheen, and A. Forryan, 2014: Suppression of internal wave breaking in the antarctic circumpolar current near topography. Journal of physical oceanography, 44 (5), 1466-1492.

Whalen, C., L. Talley, and J. MacKinnon, 2012: Spatial and temporal variability of global ocean mixing inferred from argo profiles. Geophysical Research Letters, 39 (18).

Winters, K. B., and L. Armi, 2012: Hydraulic control of continuously stratified flow over an obstacle. Journal of fluid mechanics, 700, 502-513.

Wunsch, C., and R. Ferrari, 2004: Vertical mixing, energy, and the general circulation of the oceans. Annu. Rev. Fluid Mech., 36, 281-314.

Yang, L., M. Nikurashin, A. M. Hogg, and B. M. Sloyan, 2018: Energy loss from transient eddies due to lee wave generation in the southern ocean. Journal of Physical Oceanography, 48 (12), $2867-2885$.

Zemskova, V. E., B. L. White, and A. Scotti, 2015: Available potential energy and the general circulation: Partitioning wind, buoyancy forcing, and diapycnal mixing. Journal of Physical Oceanography, 45 (6), 1510-1531.

Zheng, K., and M. Nikurashin, 2019: Downstream propagation and remote dissipation of internal waves in the southern ocean. Journal of Physical Oceanography, 49 (7), 1873-1887. 
${ }_{908}$ LIST OF TABLES

Table 1. Controlling parameters $(\chi, J)$ as defined in Eqn. (5), domain size and resolution, and maximum grid spacing normalized by Kolmogorov scale $\eta_{K}$.

44 
TABLE 1. Controlling parameters $(\chi, J)$ as defined in Eqn. (5), domain size and resolution, and maximum grid spacing normalized by Kolmogorov scale $\eta_{K}$.

\begin{tabular}{|c|c|c|c|}
\hline \hline$(\chi, J)$ & {$\left[L_{x}, L_{y}, L_{z}\right]$} & {$\left[N_{x}, N_{y}, N_{z}\right]$} & {$\left[\Delta_{x}, \Delta_{y}, \Delta_{z}\right] \max / \pi \eta_{K}$} \\
\hline$(0.16,0.6),(0.16,1),(0.16,2)$ & {$[0.5,0.5,1]$} & {$[256,256,512]$} & $(0.54,0.84,0.91)$ \\
\hline$(1.12,0.4),(1.12,0.6),(1.12,1)$ & {$[0.07,0.07,0.5]$} & {$[80,80,256]$} & $(0.83,0.88,0.93)$ \\
\hline$(0.063,0.6),(0.063,2),(0.063,5)$, & {$[1.25,0.5,1]$} & {$[640,256,512]$} & $(0.45,0.81,0.95)$ \\
\hline
\end{tabular}




\section{LIST OF FIGURES}

Fig. 1. Typical domain set-up for all simulations with sinusoidal topographic bump (upper part of the domain cropped). Overlayed are snapshots of the flow for experiment $(\chi, J)=$ $(0.16,2)$, corresponding to $\xi=0.1$, over an inertial period: (a) $t=6.25 t_{I}$, (b) $t=6.5 t_{I}$, (c) $t=6.75 t_{I}$, and (d) $t=7 t_{I}$. Color: normalized perturbation velocity $u / U$; black contours: isopycnals. The temporal progression shows an accelerated layer forming downstream of the topography, internal wave breaking, and an inertial wave propagating upward. Topography is homogeneous in $y$ and the domain is periodic in $x$ and $y$.

Fig. 2. Nondimensional parameter regimes $[J, \chi]$ for the conducted numerical simulations. Each simulation is represented with a black dot, and the contours are drawn for the topographic slope $\xi=k h_{0} / \pi$. Dashed lines indicate the extent of the lee wave radiative regime $0.1<\chi<1$.

Fig. 3. Hovmöller diagram of the normalized horizontally averaged total kinetic energy dissipation $\log _{10}\left(D\left(E_{K}\right) / U^{2}\right)$ plotted in terms of height above the bottom (HAB) for all simulations: (a,d,g) $\chi=0.063$, (b,e,h) $\chi=0.16$, (c,f,i) $\chi=1.12$. Topographic height (i.e. $J$ ) increases from the top to the bottom panels for each $\chi$. Note that the colorbar is different for $(\mathrm{a}, \mathrm{c}, \mathrm{f}, \mathrm{i})$, and that $(\mathrm{b}, \mathrm{c})$ are plotted for $t \in\left[4 t_{I}, 12 t_{I}\right]$ to demonstrate the inertial periodicity in $D\left(E_{K}\right)$, which develops at a later time than in simulations with greater $J$.

Fig. 4. (a) Temporal spectra over the last $4 t_{I}$ of total kinetic energy dissipation $D\left(E_{K}\right)$ plotted at $\mathrm{HAB}=0.1$, (b) at $\mathrm{HAB}=0.2$, and (c) horizontally integrated $D\left(E_{K}\right)$ averaged over the last $4 t_{I}$. In $(\mathrm{a}, \mathrm{b})$, spectral values are staggered for each topographic regime cluster for visual clarity. Three different topographic regimes are presented as wide topography $\chi=0.063$ in red, radiative regime $\chi=0.16$ in black, and narrow topography $\chi=1.12$ in blue. Topographic heights (i.e., $J$ ) are distinguished in increasing order as dashed, dash-dot, and solid lines.

Fig. 5. (Top) Hovmöller diagram of horizontally averaged normalized perturbation velocity $u / U$ and (bottom) time frequency spectra of $u / U$ computed over the last $4 t_{I}$ and then horizontally averaged at each depth $z$ for: $(\mathrm{a}, \mathrm{d})$ wide $\chi=0.063(\mathrm{~b}, \mathrm{e})$ radiative $\chi=0.16$, and $(\mathrm{c}, \mathrm{f})$ narrow $\chi=1.12$ topographies all with $J=0.6$.

Fig. 6. (Top) Rotary spectra for $u+i v$ over the last $4 t_{I}$ divided into clockwise (CW) and counterclockwise (CCW) components. (Bottom) Rotary bispectra, where horizontal and vertical axes are the frequencies of the first and second waves of a triad $\left(\omega_{1}, \omega_{2}\right)$, respectively, and the diagonal axis is the third sum frequency $\omega_{3}=-\left(\omega_{1}+\omega_{2}\right)$. Dashed lines indicate $\omega= \pm f, \pm 2 f$. All values are horizontally averaged at $\mathrm{HAB}=0.1$ (Left, middle, right) same as Fig. 5.

Fig. 7. (Left) Hovmöller diagram of horizontally averaged normalized perturbation velocity $u / U$, (center) rotary spectra for $u+i v$ over the last $4 t_{I}$ divided into clockwise $(\mathrm{CW})$ and counterclockwise (CCW) components, and (right) rotary bispectra. Top panels are for the simulation with $\chi=0.063, J=2$, and bottom panels for $\chi=0.063, J=5$. Rotary spectra and bispectra are horizontally averaged at $\mathrm{HAB}=0.1$.

Fig. 8. (Top) CD-filtered $u$ at frequencies (a) $\omega=0$, (b) $f$, and (c) $2 f$, as defined in Eqn. (9). (bottom) Non-linear forcing for $f$-frequency motion all defined in Eqn. (11): (d) $\Lambda_{f}$, (e) $\Lambda_{f}^{(0)}$, (f) $\Lambda_{f}^{(1)}$, (g) $\Lambda_{f}^{(0)}+\Lambda_{f}^{(1)}$. Results are for $\chi=0.16, J=0.6$ with CD-filtering over the last $4 t_{I}$. The black dashed line in (b) indicates the freely propagating inertial wave slope $\alpha_{2}$ defined in Eqn. (10). 
Fig. 9. (a,d) CD-filtered $U_{0} / U$, (b,e) CD-filtered $U_{f} / U$ (both defined in Eqn. 9), (c,f) non-linear interaction between zero- and inertial frequency motions $\Lambda_{f}^{(0)}$ defined in Eqn. (11). (a,b,c) $\chi=0.16, J=1,(\mathrm{~d}, \mathrm{e}, \mathrm{f}) \chi=0.16, J=2$. For $(\mathrm{c}, \mathrm{f})$, panels below each subfigure are vertical averages over $z \in[0,0.5]$ and panels to the right are horizontal averages of the non-linear forcing $\Lambda_{f}^{(0)}$.

Fig. 10. (Top) Snapshot of streamlines with color indicating normalized horizontal velocity $u / U$, topography marked in dashed red and LOTS in solid black lines. (Bottom) Snapshots of Ri with critical values shaded in red and overturning values in blue. (a,d) $\chi=0.16, J=0.6$, (b,e) $\chi=0.16, J=1$, and (c,f) $\chi=0.16, J=2$. All values are computed at at $t=6.5 t_{I}$.

Fig. 11. (Top) CD-filtered $u$ at $f$-frequency defined in Eqn. (9), (middle) non-linear forcing $\Lambda_{f}$ for $f$-frequency motion defined in Eqn. (11) with vertically averaged values over $z \in[0,0.4]$ below, and (bottom) time frequency spectra of $u / U$ horizontally averaged at $\mathrm{HAB}=0.05,0.1,0.2$ for the narrow $(\chi=1.12)$ topographic regime. $(\mathrm{a}, \mathrm{d}, \mathrm{g}) J=0.4$, (b,e,h) $J=0.6$, (c,f,i) $J=1$. CD-filtering and frequency spectra are both computed over last $4 t_{I}$.

Fig. 12. Snapshot of streamlines with color indicating normalized horizontal velocity $u / U$, topography marked in dashed red and LOTS in solid black lines: (a) $\chi=1.12, J=0.4$, (b) $\chi=1.12, J=0.6$, (c) $\chi=1.12, J=1$. All values are computed at $t=6.5 t_{I}$.

Fig. 13. Inertial harmonics in the wide $(\chi=0.063)$ topographic regime, and their forcing. (Left) CDfiltered $U_{f} / U$ (defined in Eqn. (9)) and (right) non-linear forcing $\Lambda_{f}$ for $f$-frequency motion defined in Eqn. (11). (a,b) $\chi=0.063, J=0.6$, (c,d) $\chi=0.063, J=2$, (e,f) $\chi=0.063, J=2$. In $(\mathrm{a}, \mathrm{c}, \mathrm{e})$, dashed lines correspond to the freely propagating inertial wave slope $\alpha_{2}$ defined in Eqn. (10). For (b,d,f), panels below each subfigure are vertical averages over $z \in[0,0.5]$ and panels to the right are horizontal averages of the non-linear forcing $\Lambda_{f}$.

Fig. 14. Same as Fig. 10 only for $(\mathrm{a}, \mathrm{d}) \chi=0.063, J=0.6,(\mathrm{~b}, \mathrm{e}) \chi=0.063, J=2,(\mathrm{c}, \mathrm{f}) \chi=0.063, J=5$. All values are computed at $t=6.5 t_{I}$.

Fig. A1. Comparison of horizontally-averaged profiles between runs with and without stratification restoration in $\chi=0.16, J=2$ regime: (a) buoyancy averaged over last $t_{I}$ compared with background stratification $B(z)$, (b) velocity $u / U$ averaged over last $t_{I}$, (c) kinetic energy dissipation $D\left(E_{K}\right)$ averaged over last $4 t_{I}$. 
(a)

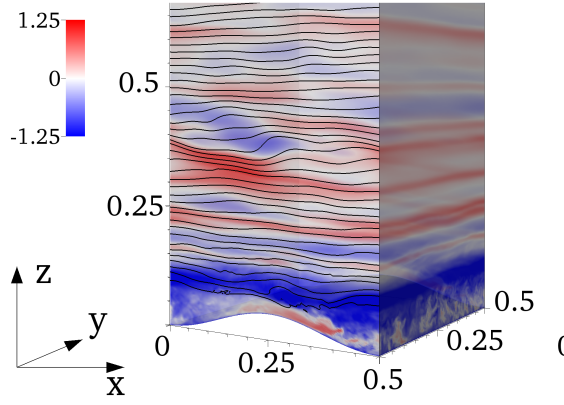

(b)

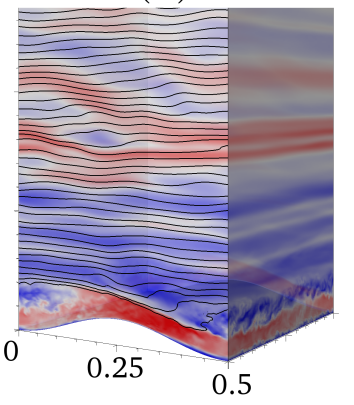

(c)

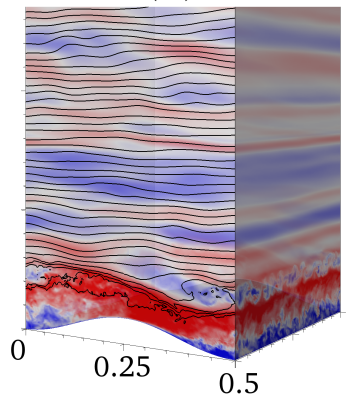

(d)

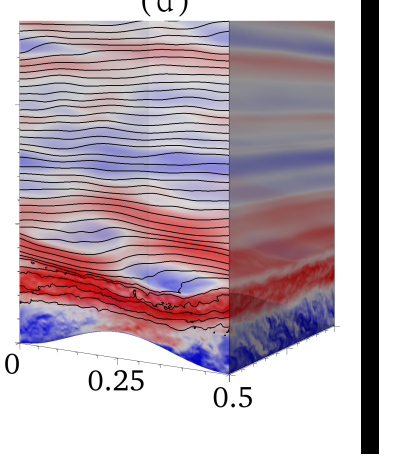

FIG. 1. Typical domain set-up for all simulations with sinusoidal topographic bump (upper part of the domain cropped). Overlayed are snapshots of the flow for experiment $(\chi, J)=(0.16,2)$, corresponding to $\xi=0.1$, over an inertial period: (a) $t=6.25 t_{I}$, (b) $t=6.5 t_{I}$, (c) $t=6.75 t_{I}$, and (d) $t=7 t_{I}$. Color: normalized perturbation velocity $u / U$; black contours: isopycnals. The temporal progression shows an accelerated layer forming downstream of the topography, internal wave breaking, and an inertial wave propagating upward. Topography is homogeneous in $y$ and the domain is periodic in $x$ and $y$. 


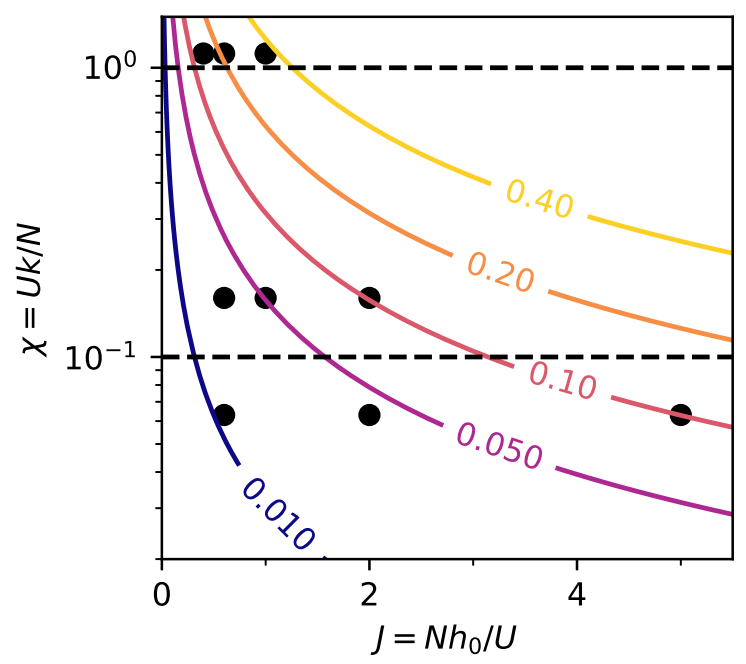

FIG. 2. Nondimensional parameter regimes $[J, \chi]$ for the conducted numerical simulations. Each simulation is represented with a black dot, and the contours are drawn for the topographic slope $\xi=k h_{0} / \pi$. Dashed lines indicate the extent of the lee wave radiative regime $0.1<\chi<1$. 
(a) $\chi=0.063, J=0.6$

(b) $\chi=0.16, J=0.6$
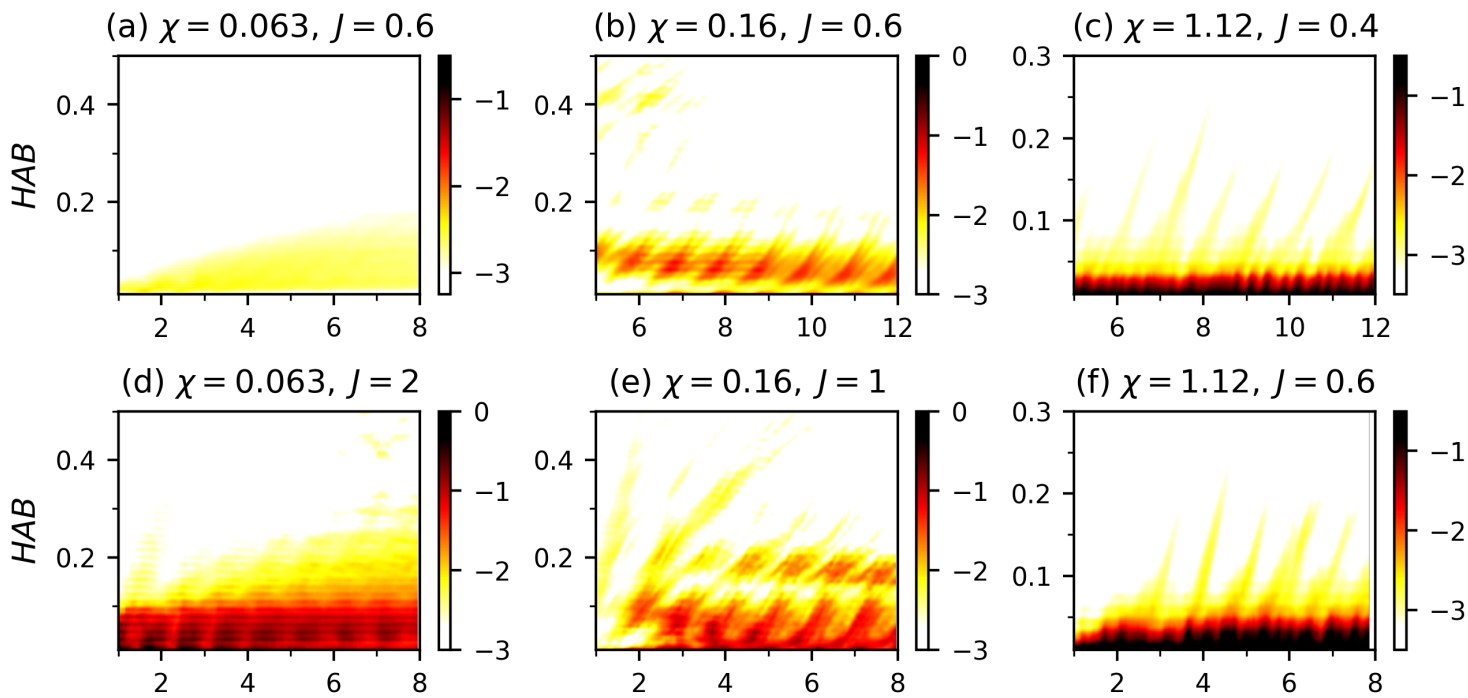

(e) $\chi=0.16, J=1$
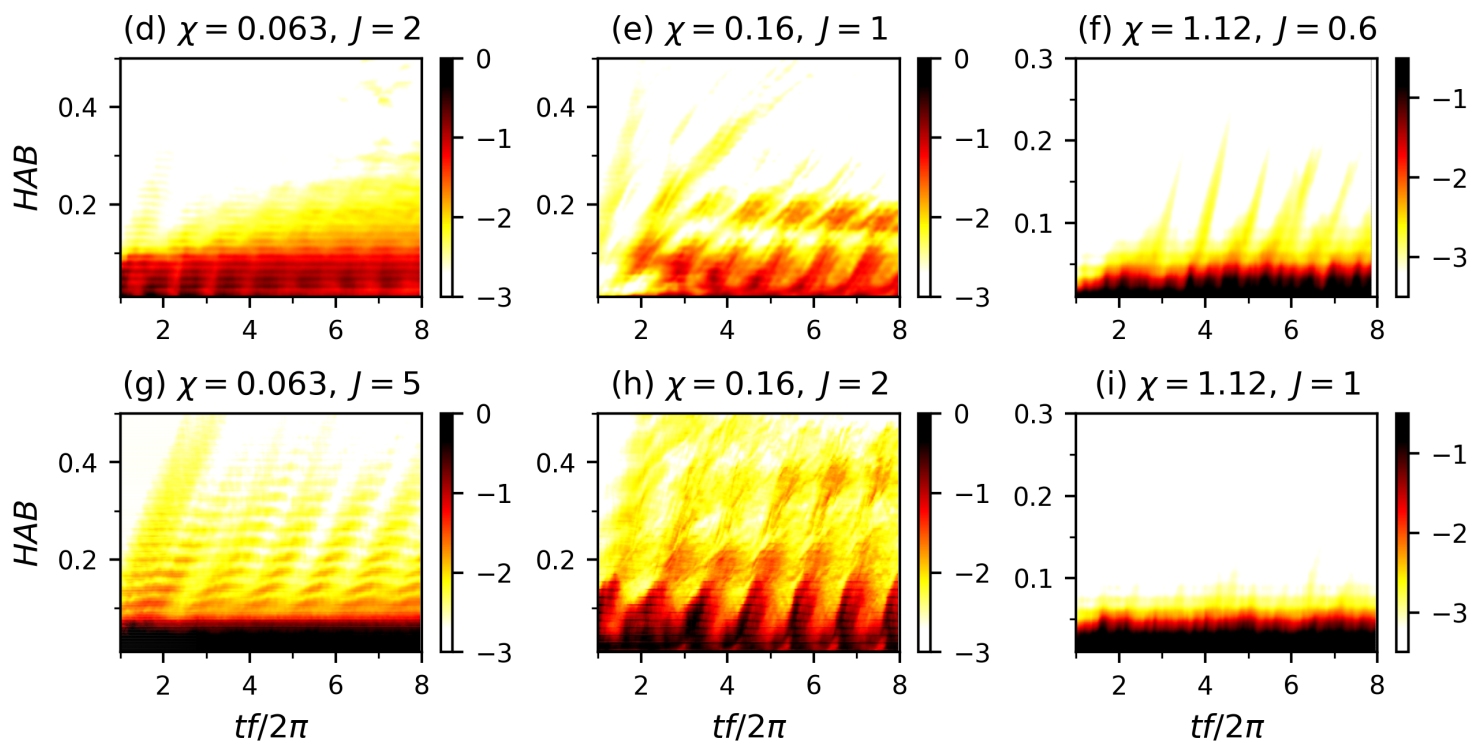

FIG. 3. Hovmöller diagram of the normalized horizontally averaged total kinetic energy dissipation $\log _{10}\left(D\left(E_{K}\right) / U^{2}\right)$ plotted in terms of height above the bottom (HAB) for all simulations: $(\mathrm{a}, \mathrm{d}, \mathrm{g}) \chi=0.063$, $(\mathrm{b}, \mathrm{e}, \mathrm{h}) \chi=0.16$, (c,f,i) $\chi=1.12$. Topographic height (i.e. $J$ ) increases from the top to the bottom panels for each $\chi$. Note that the colorbar is different for $(\mathrm{a}, \mathrm{c}, \mathrm{f}, \mathrm{i})$, and that $(\mathrm{b}, \mathrm{c})$ are plotted for $t \in\left[4 t_{I}, 12 t_{I}\right]$ to demonstrate the inertial periodicity in $D\left(E_{K}\right)$, which develops at a later time than in simulations with greater $J$. 
(a) $H A B=0.1$

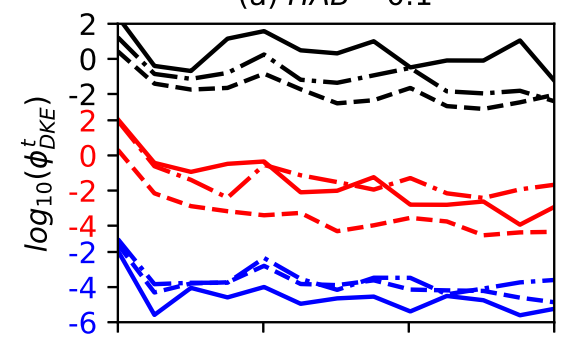

(b) $H A B=0.2$

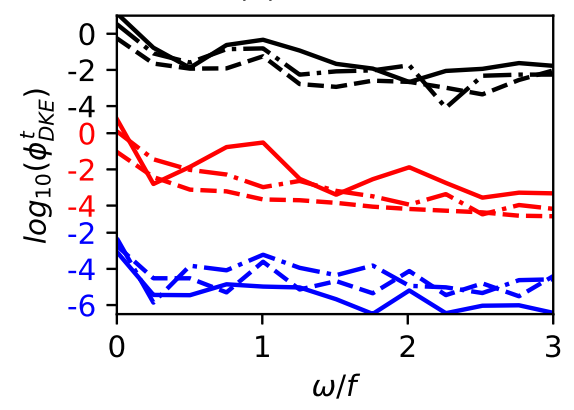

(c)

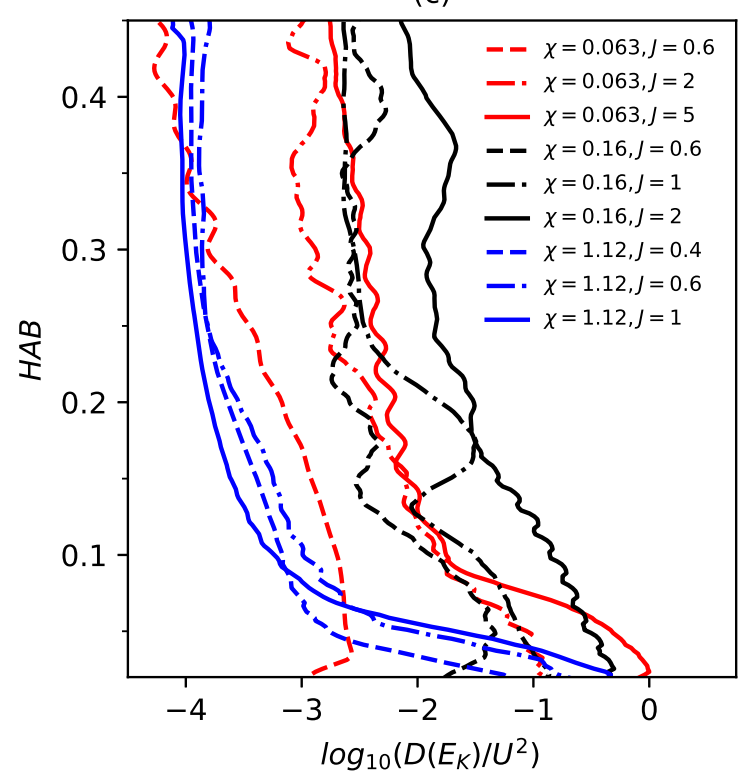

FIG. 4. (a) Temporal spectra over the last $4 t_{I}$ of total kinetic energy dissipation $D\left(E_{K}\right)$ plotted at $\mathrm{HAB}=0.1$, (b) at $\mathrm{HAB}=0.2$, and (c) horizontally integrated $D\left(E_{K}\right)$ averaged over the last $4 t_{I}$. In (a,b), spectral values are staggered for each topographic regime cluster for visual clarity. Three different topographic regimes are presented as wide topography $\chi=0.063$ in red, radiative regime $\chi=0.16$ in black, and narrow topography $\chi=1.12$ in blue. Topographic heights (i.e., $J$ ) are distinguished in increasing order as dashed, dash-dot, and solid lines. 

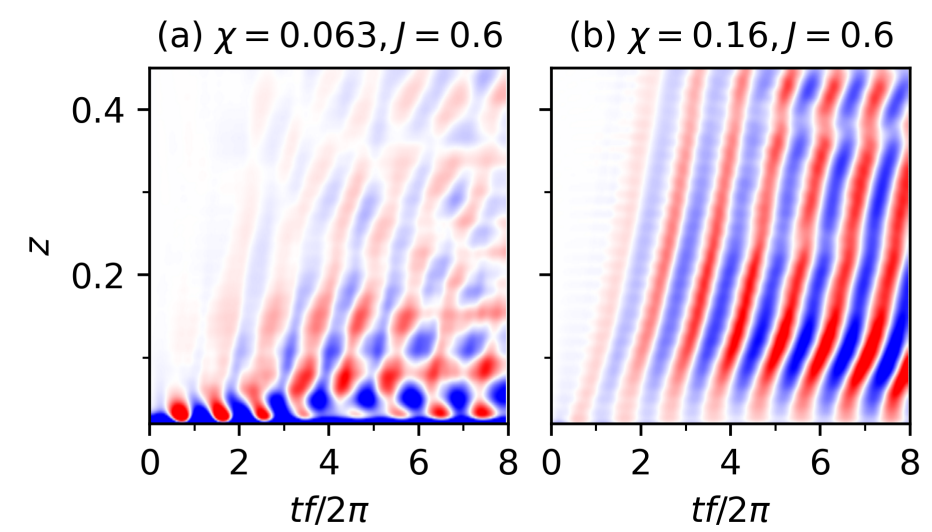

(c) $\chi=1.12, J=0.6$
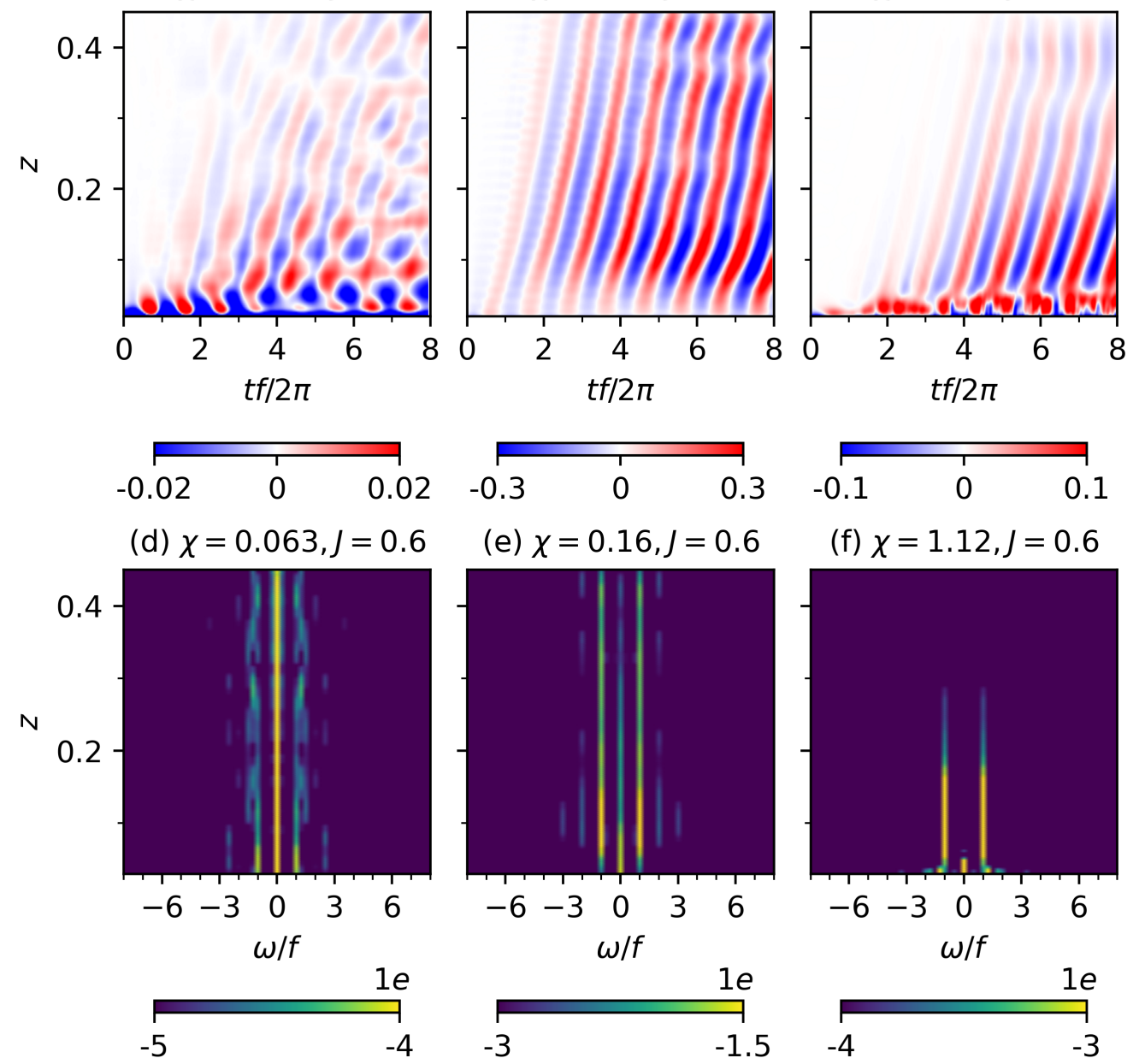

FIG. 5. (Top) Hovmöller diagram of horizontally averaged normalized perturbation velocity $u / U$ and (bottom) time frequency spectra of $u / U$ computed over the last $4 t_{I}$ and then horizontally averaged at each depth $z$ for: $(\mathrm{a}, \mathrm{d})$ wide $\chi=0.063(\mathrm{~b}, \mathrm{e})$ radiative $\chi=0.16$, and $(\mathrm{c}, \mathrm{f})$ narrow $\chi=1.12$ topographies all with $J=0.6$ 

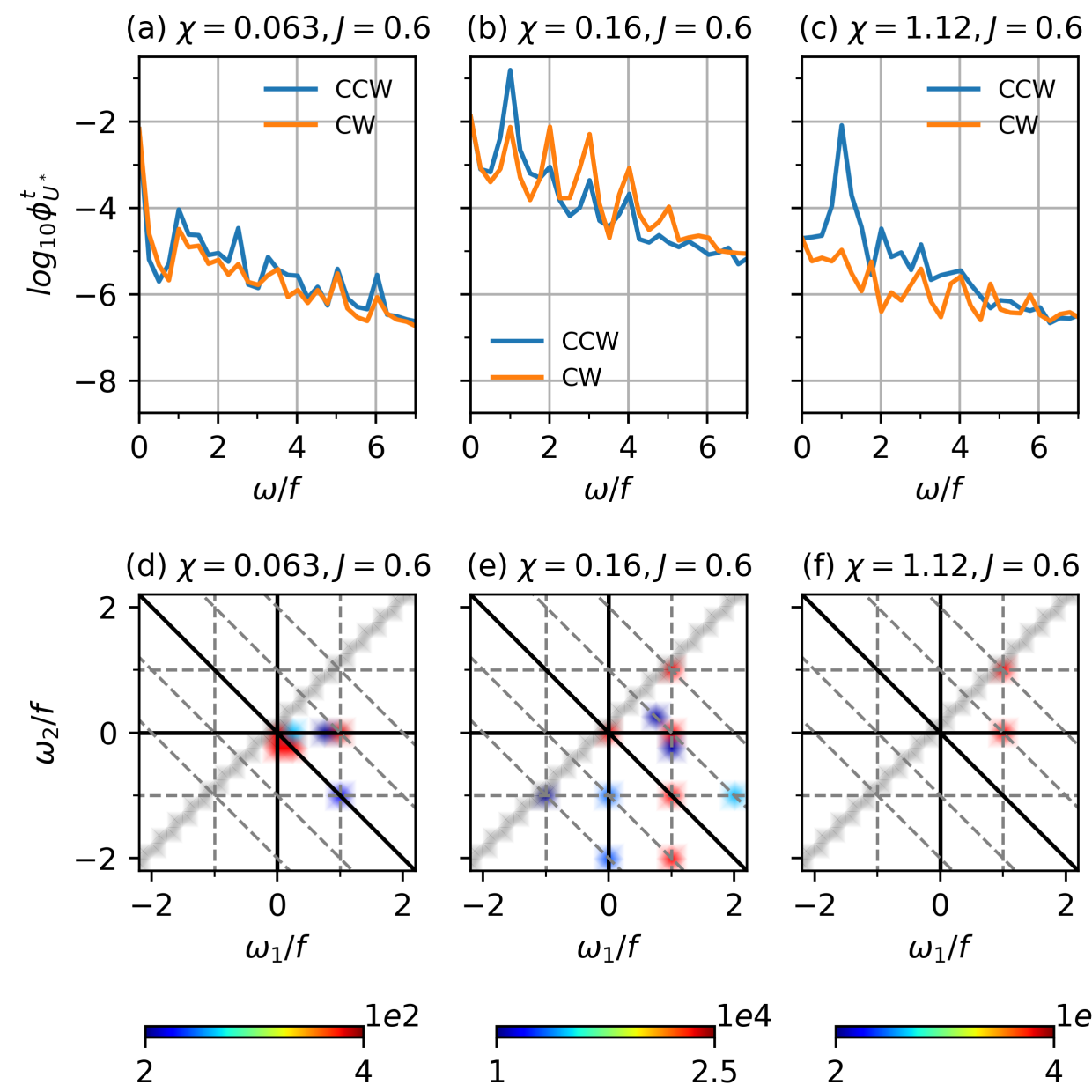

FIG. 6. (Top) Rotary spectra for $u+i v$ over the last $4 t_{I}$ divided into clockwise $(\mathrm{CW})$ and counterclockwise (CCW) components. (Bottom) Rotary bispectra, where horizontal and vertical axes are the frequencies of the first and second waves of a triad $\left(\omega_{1}, \omega_{2}\right)$, respectively, and the diagonal axis is the third sum frequency $\omega_{3}=-\left(\omega_{1}+\omega_{2}\right)$. Dashed lines indicate $\omega= \pm f, \pm 2 f$. All values are horizontally averaged at HAB $=0.1$ (Left, middle, right) same as Fig. 5. 

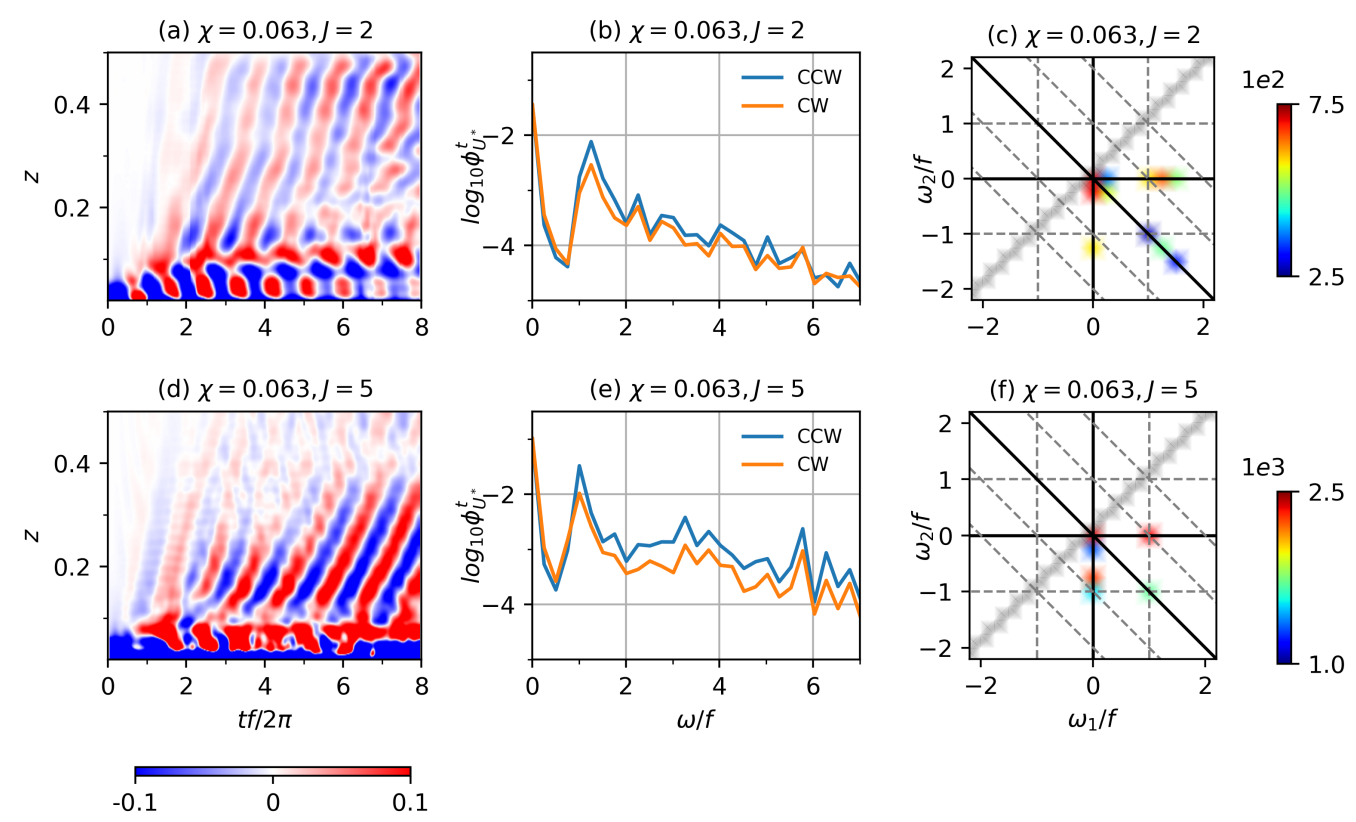

FIG. 7. (Left) Hovmöller diagram of horizontally averaged normalized perturbation velocity $u / U$, (center) rotary spectra for $u+i v$ over the last $4 t_{I}$ divided into clockwise $(\mathrm{CW})$ and counterclockwise $(\mathrm{CCW})$ components, and (right) rotary bispectra. Top panels are for the simulation with $\chi=0.063, J=2$, and bottom panels for 1016 $\chi=0.063, J=5$. Rotary spectra and bispectra are horizontally averaged at $\mathrm{HAB}=0.1$. 
(a) $U_{0} / U$

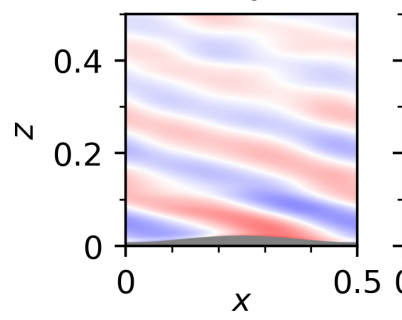

(b) $U_{f} / U$

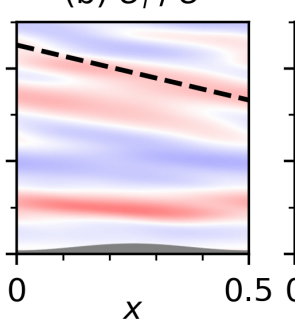

(c) $U_{2 f} / U$

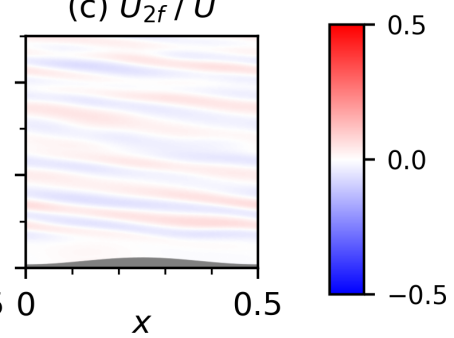

(d) $\wedge_{f}$

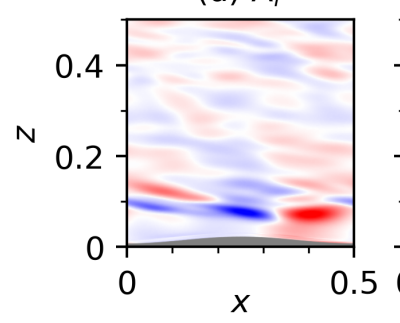

(e) $\wedge_{f}^{(0)}$

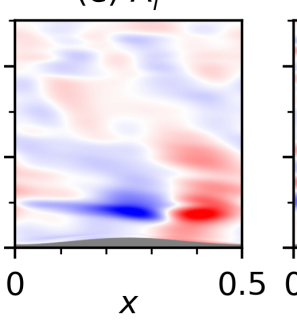

(f) $\Lambda_{f}^{(1)}$

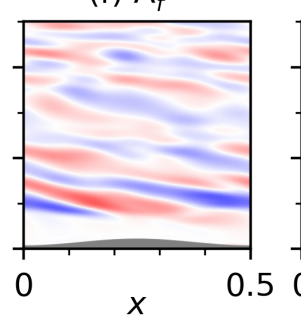

(g) $\Lambda_{f}^{(0)}+\Lambda_{f}^{(1)}$

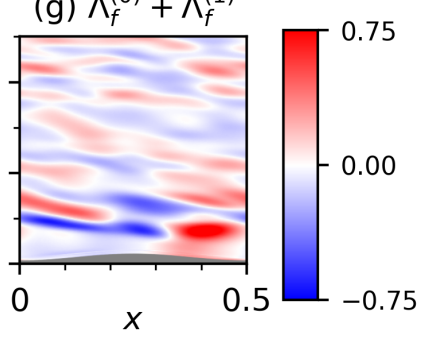

FIg. 8. (Top) CD-filtered $u$ at frequencies (a) $\omega=0$, (b) $f$, and (c) $2 f$, as defined in Eqn. (9). (bottom) Non-linear forcing for $f$-frequency motion all defined in Eqn. (11): (d) $\Lambda_{f}$, (e) $\Lambda_{f}^{(0)}$, (f) $\Lambda_{f}^{(1)},(\mathrm{g}) \Lambda_{f}^{(0)}+\Lambda_{f}^{(1)}$. Results are for $\chi=0.16, J=0.6$ with CD-filtering over the last $4 t_{I}$. The black dashed line in (b) indicates the freely propagating inertial wave slope $\alpha_{2}$ defined in Eqn. (10). 
(a) $U_{0} / U: \chi=0.16, J=1$ (b) $U_{f} / U: \chi=0.16, J=1$
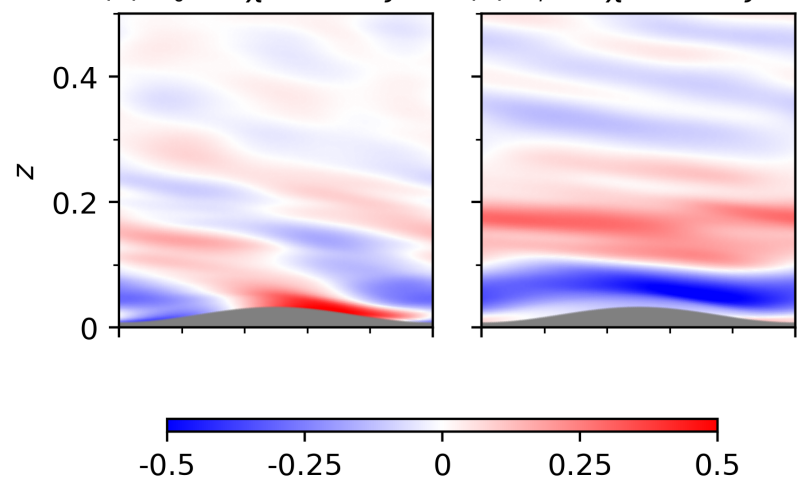

(d) $U_{0} / U: \chi=0.16, J=2$ (e) $U_{f} / U: \chi=0.16, J=2$
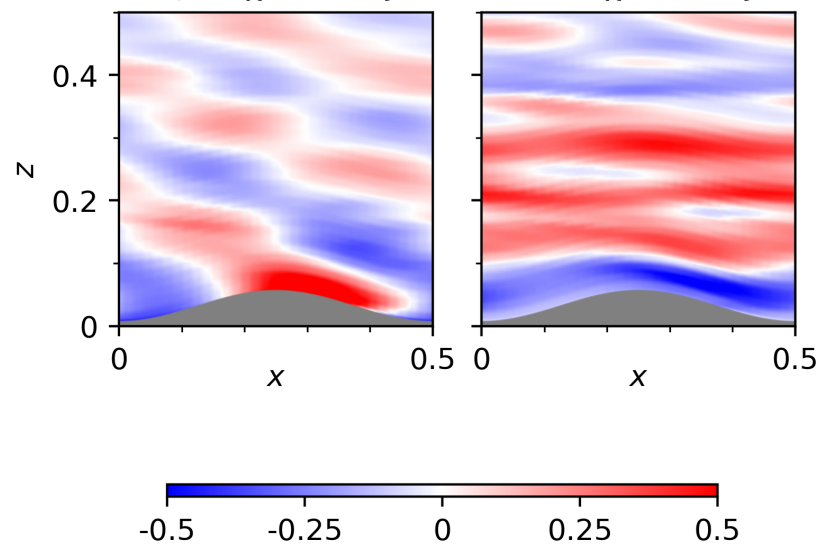

(c) $\Lambda_{f}^{(0)}: \chi=0.16, J=1$
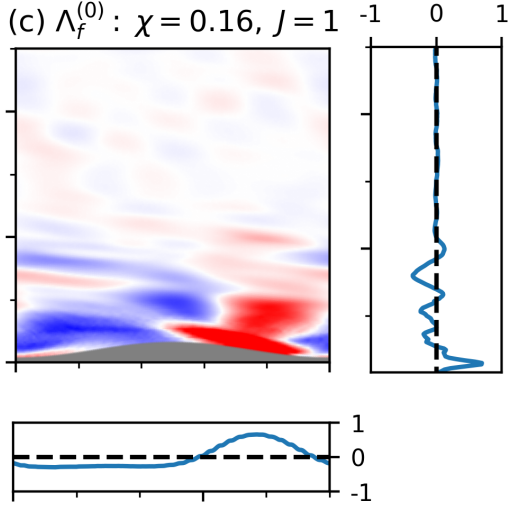

(f) $\Lambda_{f}^{(0)}: \chi=0.16, J=2 \quad-3 \quad 0 \quad 3$
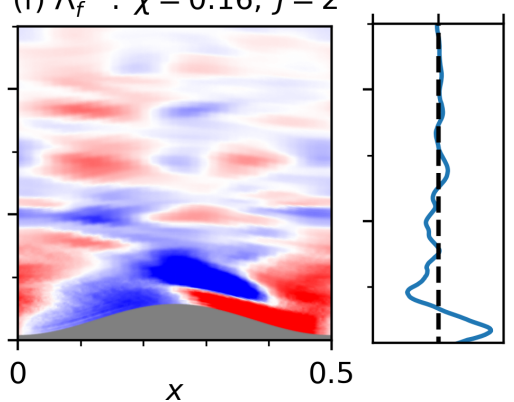

0.5

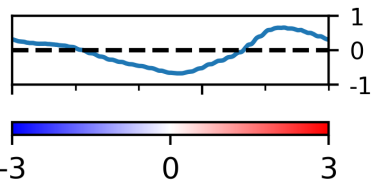

FIG. 9. (a,d) CD-filtered $U_{0} / U$, (b,e) CD-filtered $U_{f} / U$ (both defined in Eqn. 9), (c,f) non-linear interaction between zero- and inertial frequency motions $\Lambda_{f}^{(0)}$ defined in Eqn. (11). (a,b,c) $\chi=0.16, J=1$, (d,e,f) $\chi=$ $0.16, J=2$. For $(\mathrm{c}, \mathrm{f})$, panels below each subfigure are vertical averages over $z \in[0,0.5]$ and panels to the right are horizontal averages of the non-linear forcing $\Lambda_{f}^{(0)}$. 

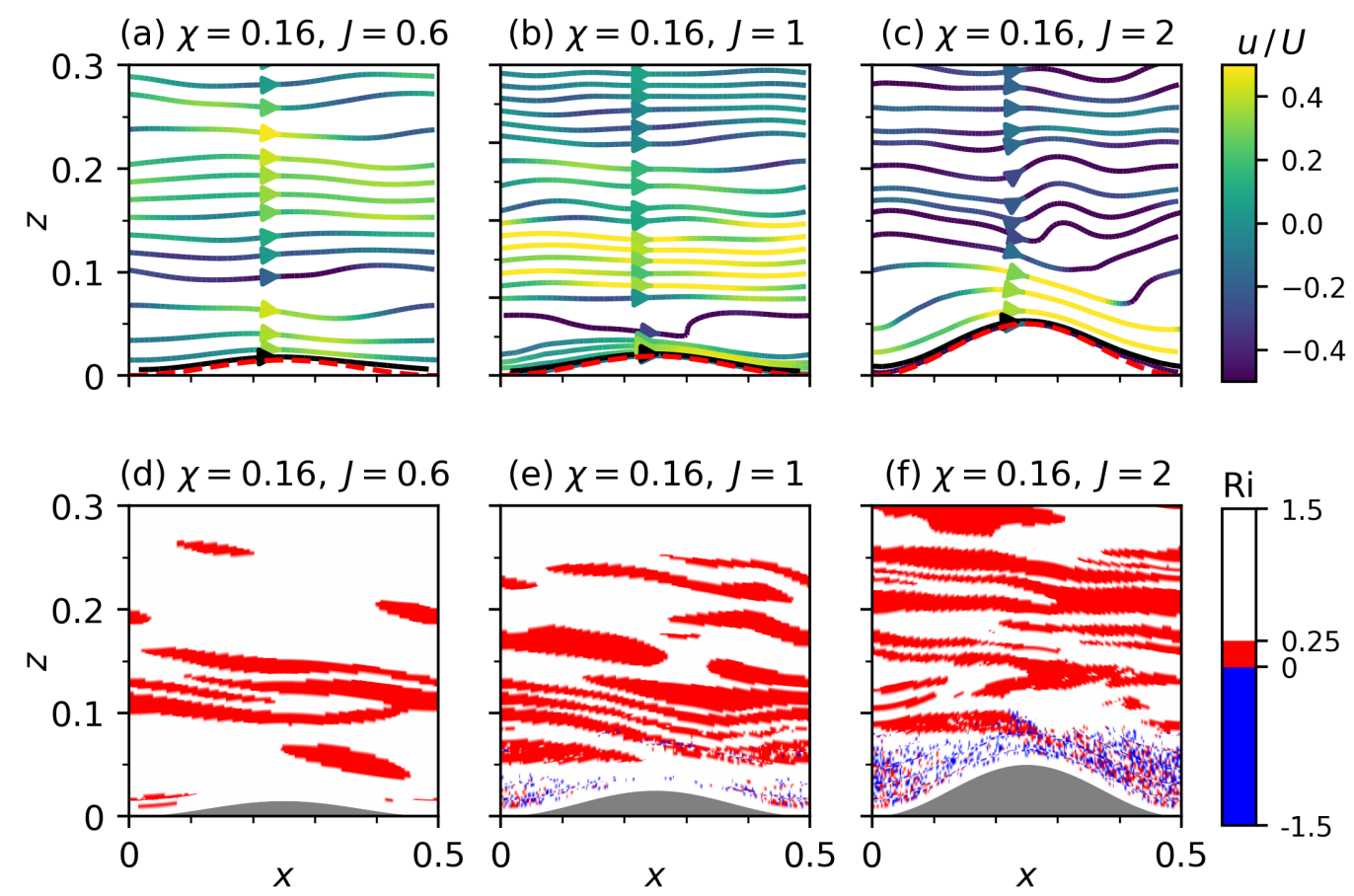

(e) $\chi=0.16, J=1$
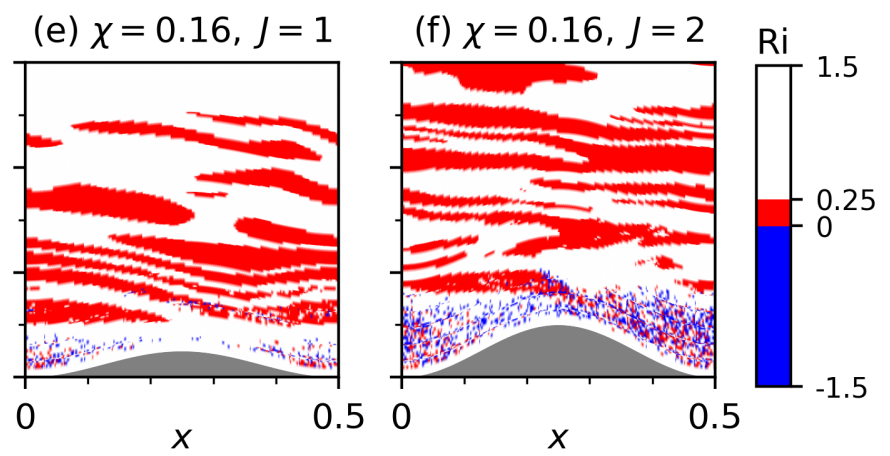

FIG. 10. (Top) Snapshot of streamlines with color indicating normalized horizontal velocity $u / U$, topography marked in dashed red and LOTS in solid black lines. (Bottom) Snapshots of Ri with critical values shaded in red and overturning values in blue. (a,d) $\chi=0.16, J=0.6$, (b,e) $\chi=0.16, J=1$, and (c,f) $\chi=0.16, J=2$. All values are computed at at $t=6.5 t_{I}$. 
(a) $U_{f} / U$ :

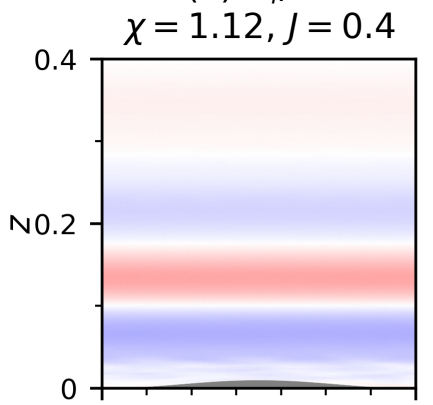

(b) $U_{f} / U$ :
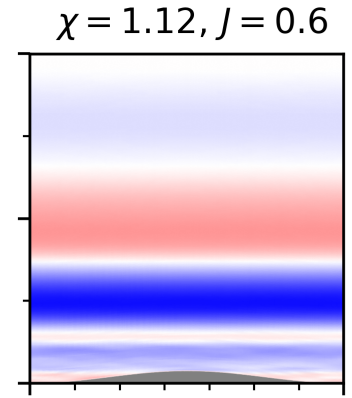

(c) $U_{f} / U$ :

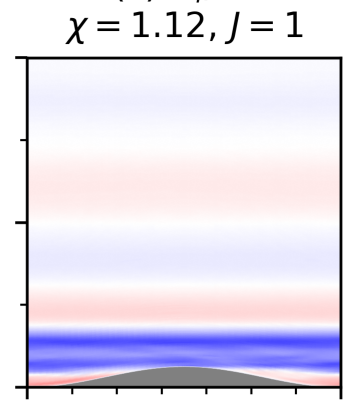

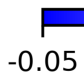

0.05

(d) $\wedge_{f}: \chi=1.12, J=0.4$

(e) $\wedge_{f}: \chi=1.12, J=0.6$

(f) $\wedge_{f}: \chi=1.12, J=1$
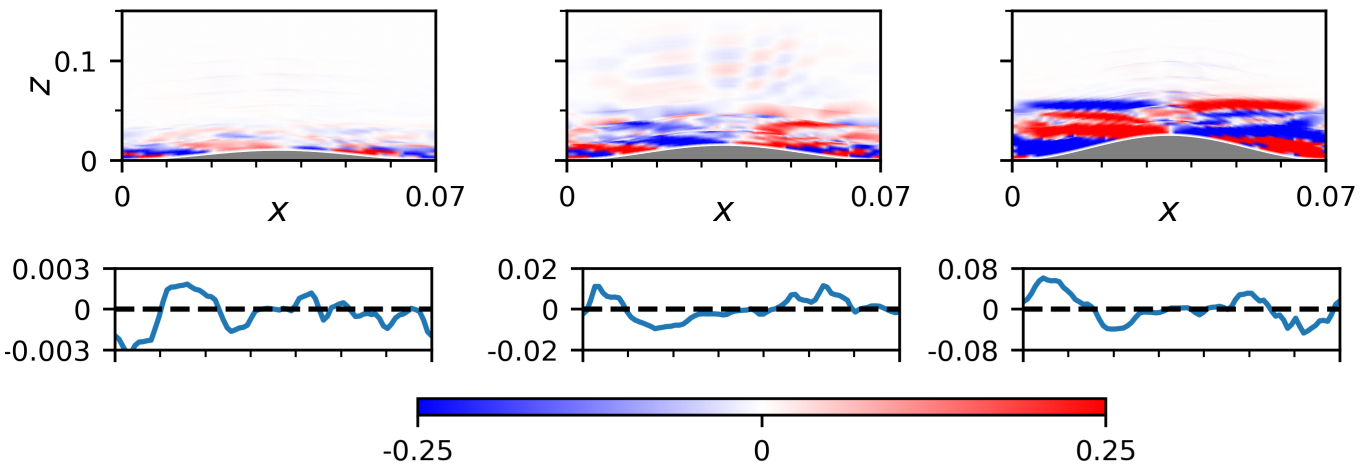

(i) $\chi=1.12, J=1$

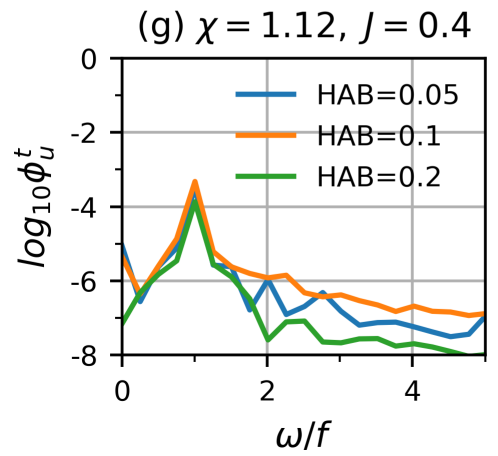

(h) $\chi=1.12, J=0.6$

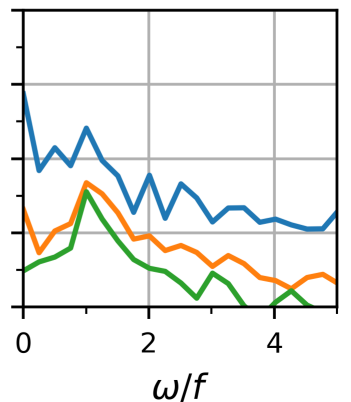

FIG. 11. (Top) CD-filtered $u$ at $f$-frequency defined in Eqn. (9), (middle) non-linear forcing $\Lambda_{f}$ for $f$-frequency motion defined in Eqn. (11) with vertically averaged values over $z \in[0,0.4]$ below, and (bottom) time frequency spectra of $u / U$ horizontally averaged at $\mathrm{HAB}=0.05,0.1,0.2$ for the narrow $(\chi=1.12)$ topographic regime. (a,d,g) $J=0.4,(\mathrm{~b}, \mathrm{e}, \mathrm{h}) J=0.6,(\mathrm{c}, \mathrm{f}, \mathrm{i}) J=1$. CD-filtering and frequency spectra are both computed over last $4 t_{I}$. 

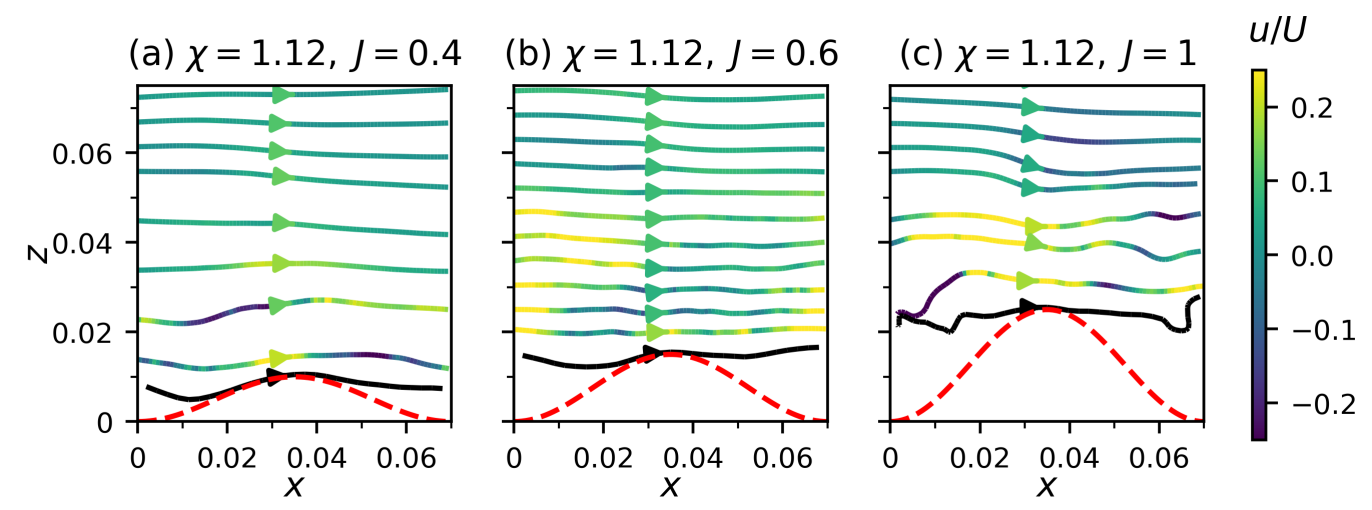

FIG. 12. Snapshot of streamlines with color indicating normalized horizontal velocity $u / U$, topography marked in dashed red and LOTS in solid black lines: (a) $\chi=1.12, J=0.4$, (b) $\chi=1.12, J=0.6$, (c) $\chi=1.12, J=1$. All values are computed at $t=6.5 t_{I}$. 
(a) $U_{f} / U: \chi=0.063, J=0.6$

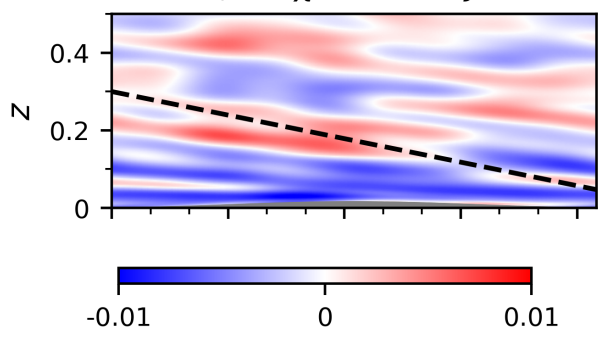

(c) $U_{f} / U: \chi=0.063, J=2$
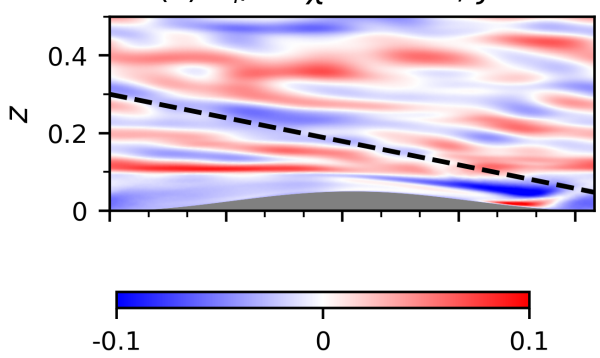

(e) $U_{f} / U: \chi=0.063, J=5$

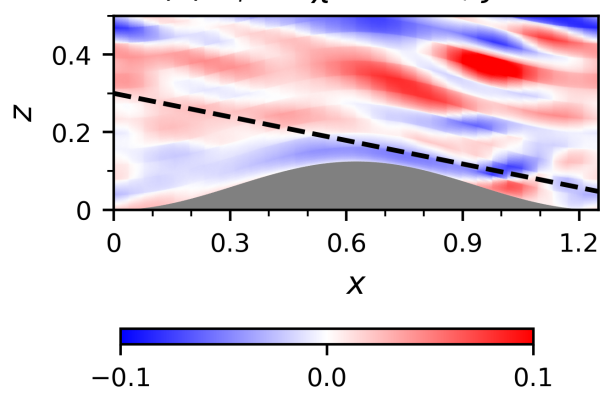

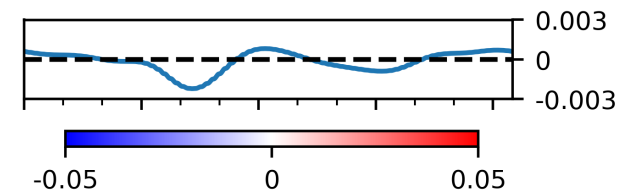

(b) $\wedge_{f}: \chi=0.063, J=0.6$

$\begin{array}{lll}0.01 & 0 & 0.01\end{array}$

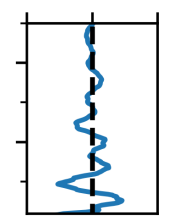

0.003

$-003$

(d) $\wedge_{f}: \chi=0.063, J=2$
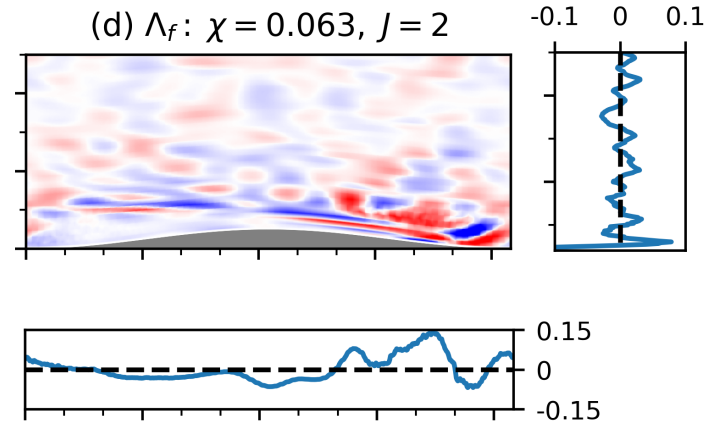

(f) $\Lambda_{f}: \chi=0.063, J=5$

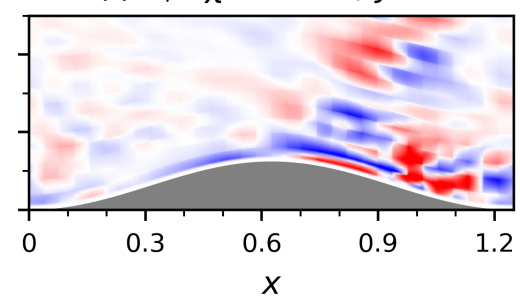

$\begin{array}{lll}0.15 & 0 & 0.15\end{array}$

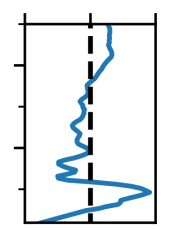

0.15

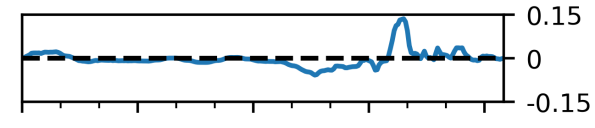

$-1$

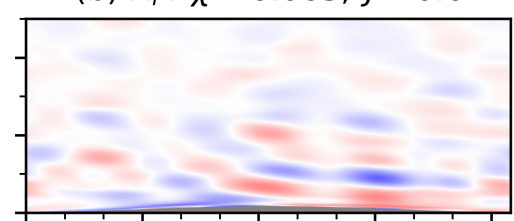

FIG. 13. Inertial harmonics in the wide $(\chi=0.063)$ topographic regime, and their forcing. (Left) CD-filtered $U_{f} / U$ (defined in Eqn. (9)) and (right) non-linear forcing $\Lambda_{f}$ for $f$-frequency motion defined in Eqn. (11). (a,b) $\chi=0.063, J=0.6,(\mathrm{c}, \mathrm{d}) \chi=0.063, J=2,(\mathrm{e}, \mathrm{f}) \chi=0.063, J=2$. In (a,c,e), dashed lines correspond to the freely propagating inertial wave slope $\alpha_{2}$ defined in Eqn. (10). For (b,d,f), panels below each subfigure are vertical averages over $z \in[0,0.5]$ and panels to the right are horizontal averages of the non-linear forcing $\Lambda_{f}$. 


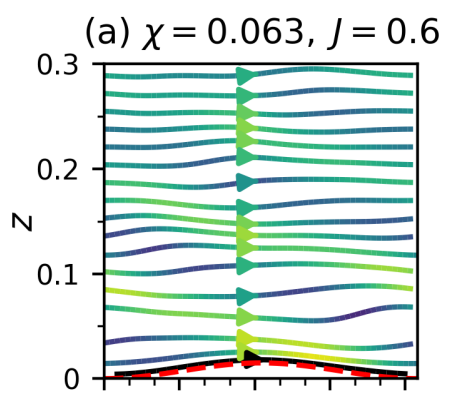

(b) $\chi=0.063, J=2$

(c) $\chi=0.063, J=5$
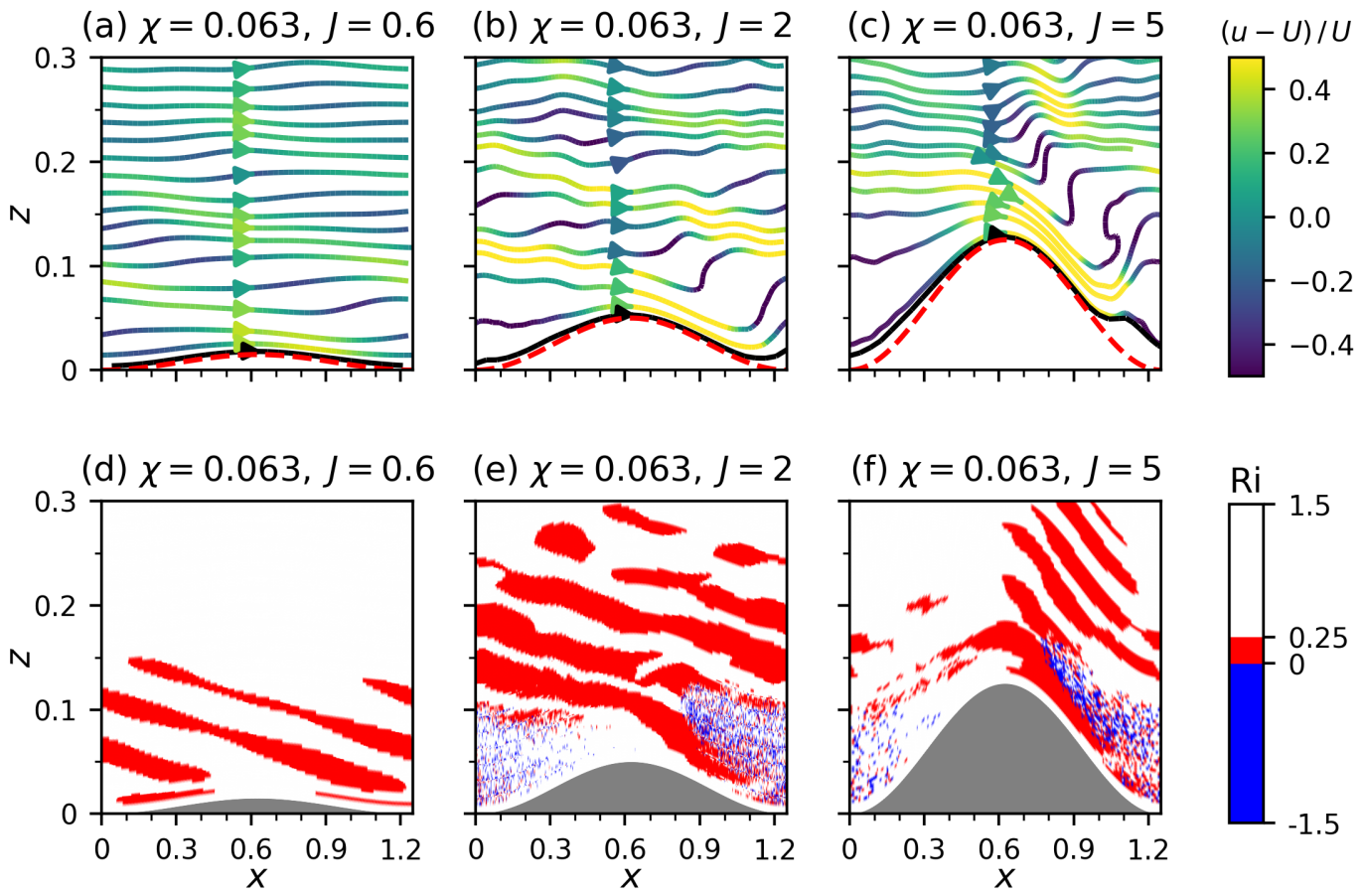

FIG. 14. Same as Fig. 10 only for (a,d) $\chi=0.063, J=0.6$, (b,e) $\chi=0.063, J=2,(\mathrm{c}, \mathrm{f}) \chi=0.063, J=5$. All 1042 values are computed at $t=6.5 t_{I}$. 
(a)

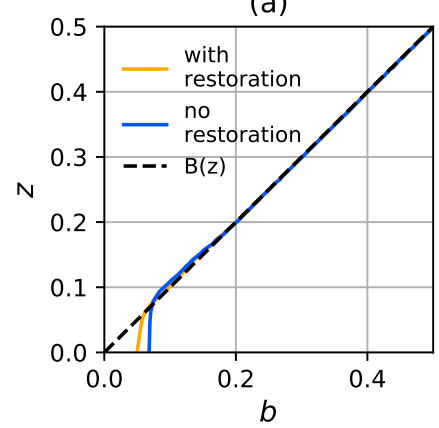

(b)

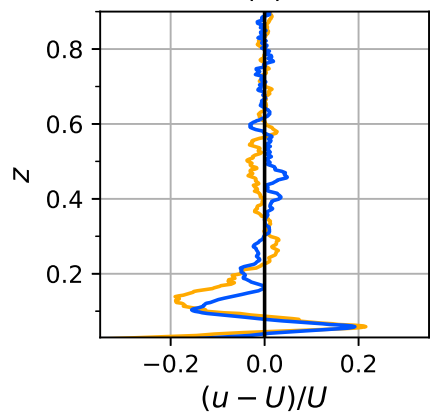

(c)

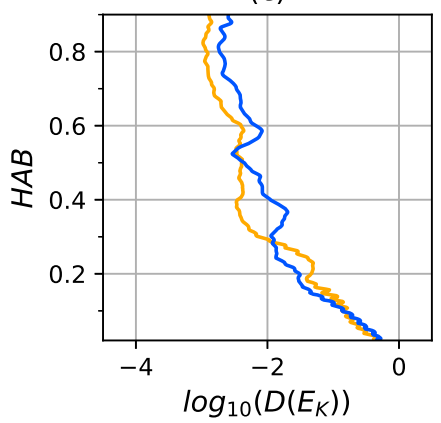

Fig. A1. Comparison of horizontally-averaged profiles between runs with and without stratification restoration in $\chi=0.16, J=2$ regime: (a) buoyancy averaged over last $t_{I}$ compared with background stratification $B(z)$, (b) velocity $u / U$ averaged over last $t_{I}$, (c) kinetic energy dissipation $D\left(E_{K}\right)$ averaged over last $4 t_{I}$. 